\title{
MARKOV DECISION PROCESSES WITH MULTIPLE LONG-RUN AVERAGE OBJECTIVES
}

\author{
TOMÁŠ BRÁZDIL ${ }^{a}$, VÁCLAV BROŽEK, KRISHNENDU CHATTERJEE $^{c}$, VOJTĚCH FOREJT $^{d}$, \\ AND ANTONÍN KUČERA ${ }^{e}$
}

${ }^{a, b, e}$ Faculty of Informatics, Masaryk University, Brno, Czech Republic

e-mail address: \{brazdil,xbrozek,kucera\}@fi.muni.cz

${ }^{c}$ IST Austria, Klosterneuburg, Austria

e-mail address: krish.chat@ist.ac.at

${ }^{d}$ Department of Computer Science, University of Oxford, UK

e-mail address: vojfor@comlab.ox.ac.uk

\begin{abstract}
We study Markov decision processes (MDPs) with multiple limit-average (or mean-payoff) functions. We consider two different objectives, namely, expectation and satisfaction objectives. Given an MDP with $k$ limit-average functions, in the expectation objective the goal is to maximize the expected limit-average value, and in the satisfaction objective the goal is to maximize the probability of runs such that the limit-average value stays above a given vector. We show that under the expectation objective, in contrast to the case of one limit-average function, both randomization and memory are necessary for strategies even for $\boldsymbol{\varepsilon}$-approximation, and that finite-memory randomized strategies are sufficient for achieving Pareto optimal values. Under the satisfaction objective, in contrast to the case of one limit-average function, infinite memory is necessary for strategies achieving a specific value (i.e. randomized finite-memory strategies are not sufficient), whereas memoryless randomized strategies are sufficient for $\boldsymbol{\varepsilon}$-approximation, for all $\boldsymbol{\varepsilon}>\mathbf{0}$. We further prove that the decision problems for both expectation and satisfaction objectives can be solved in polynomial time and the trade-off curve (Pareto curve) can be $\varepsilon$-approximated in time polynomial in the size of the MDP and $\frac{1}{\varepsilon}$, and exponential in the number of limitaverage functions, for all $\boldsymbol{\varepsilon}>\mathbf{0}$. Our analysis also reveals flaws in previous work for MDPs with multiple mean-payoff functions under the expectation objective, corrects the flaws, and allows us to obtain improved results.
\end{abstract}

2012 ACM CCS: [Mathematics of computing]: Probability and statistics-Stochastic processesMarkov processes; Design and analysis of algorithms-Mathematical optimization-Continuous optimization-Stochastic control and optimization/Convex Optimization; [Software and its engineering]: Software creation and management-Software verification and validation-Formal software verification.

Key words and phrases: Markov decision processes, mean-payoff reward, multi-objective optimisation, formal verification.

넴
DOI:10.2168/LMCS-10(1:13)2014
(C) T. Brázdil, V. Brožek, K. Chatterjee, V. Forejt, and A. Kučera (c) Creative Commons 


\section{INTRODUCTION}

Markov decision processes (MDPs) are the standard models for probabilistic dynamic systems that exhibit both probabilistic and nondeterministic behaviors [18, 11]. In each state of an MDP, a controller chooses one of several actions (the nondeterministic choices), and the system stochastically evolves to a new state based on the current state and the chosen action. A reward (or cost) is associated with each transition and the central question is to find a strategy of choosing the actions that optimizes the rewards obtained over the run of the system. One classical way to combine the rewards over the run of the system is the limit-average (or mean-payoff) function that assigns to every run the average of the rewards over the run. MDPs with single mean-payoff functions have been widely studied in literature (see, e.g., [18, 11]). In many modeling domains, however, there is not a single goal to be optimized, but multiple, potentially dependent and conflicting goals. For example, in designing a computer system, the goal is to maximize average performance while minimizing average power consumption. Similarly, in an inventory management system, the goal is to optimize several potentially dependent costs for maintaining each kind of product. These motivate the study of MDPs with multiple mean-payoff functions.

Traditionally, MDPs with mean-payoff functions have been studied with only the $e x$ pectation objective, where the goal is to maximize (or minimize) the expectation of the mean-payoff function. There are numerous applications of MDPs with expectation objectives in inventory control, planning, and performance evaluation [18, 11. In this work we consider both the expectation objective and also the satisfaction objective for a given MDP. In both cases we are given an MDP with $k$ reward functions, and the goal is to maximize (or minimize) either the $k$-tuple of expectations, or the probability of runs such that the mean-payoff value stays above a given vector.

To get some intuition about the difference between the expectation/satisfaction objectives and to show that in some scenarios the satisfaction objective is preferable, consider a filehosting system where the users can download files at various speed, depending on the current setup and the number of connected customers. For simplicity, let us assume that a user has $20 \%$ chance to get a $2000 \mathrm{kB} / \mathrm{sec}$ connection, and $80 \%$ chance to get a slow $20 \mathrm{kB} / \mathrm{sec}$ connection. Then, the overall performance of the server can be reasonably measured by the expected amount of transferred data per user and second (i.e., the expected mean payoff) which is $416 \mathrm{kB} / \mathrm{sec}$. However, a single user is more interested in her chance of downloading the files quickly, which can be measured by the probability of establishing and maintaining a reasonably fast connection (say, $\geq 1500 \mathrm{kB} / \mathrm{sec}$ ). Hence, the system administrator may want to maximize the expected mean payoff (by changing the internal setup of the system), while a single user aims at maximizing the probability of satisfying her preferences (she can achieve that, e.g., by buying a priority access, waiting till 3 a.m., or simply connecting to a different server; obviously, she might also wish to minimize other mean payoffs such as the price per transferred bit). In other words, the expectation objective is relevant in situations when we are interested in the "average" behaviour of many instances of a given system, while the satisfaction objective is useful for analyzing and optimizing particular executions.

In MDPs with multiple mean-payoff functions, various strategies may produce incomparable solutions, and consequently there is no "best" solution in general. Informally, the set of achievable solutions

(i) under the expectation objective is the set of all vectors $\vec{v}$ such that there is a strategy to ensure that the expected mean-payoff value vector under the strategy is at least $\vec{v}$; 
(ii) under the satisfaction objective is the set of tuples $(\nu, \vec{v})$ where $\nu \in[0,1]$ and $\vec{v}$ is a vector such that there is a strategy under which with probability at least $\nu$ the mean-payoff value vector of a run is at least $\vec{v}$.

The "trade-offs" among the goals represented by the individual mean-payoff functions are formally captured by the Pareto curve, which consists of all minimal tuples (wrt. componentwise ordering) that are not strictly dominated by any achievable solution. Intuitively, the Pareto curve consists of "limits" of achievable solutions, and in principle it may contain tuples that are not achievable solutions (see Section 3). Pareto optimality has been studied in cooperative game theory [16] and in multi-criterion optimization and decision making in both economics and engineering [14, 21, 20].

Our study of MDPs with multiple mean-payoff functions is motivated by the following fundamental questions, which concern both basic properties and algorithmic aspects of the expectation/satisfaction objectives:

Q.1 What type of strategies is sufficient (and necessary) for achievable solutions?

Q.2 Are the elements of the Pareto curve achievable solutions?

Q.3 Is it decidable whether a given vector represents an achievable solution?

Q.4 Given an achievable solution, is it possible to compute a strategy which achieves this solution?

Q.5 Is it decidable whether a given vector belongs to the Pareto curve?

Q.6 Is it possible to compute a finite representation/approximation of the Pareto curve?

We provide comprehensive answers to the above questions, both for the expectation and the satisfaction objective. We also analyze the complexity of the problems given in Q.3-Q.6. From a practical point of view, it is particularly encouraging that most of the considered problems turn out to be solvable efficiently, i.e., in polynomial time. More concretely, our answers to Q.1-Q.6 are the following:

1.a For the expectation objectives, finite-memory randomized strategies are sufficient and necessary for all achievable solutions. Memory and randomization may also be needed to approximate an achievable solution up to $\varepsilon$ for a given $\varepsilon>0$.

1.b For the satisfaction objectives, achievable solutions require infinite memory in general, but memoryless randomized strategies are sufficient to approximate any achievable solution up to an arbitrarily small $\varepsilon>0$.

2. All elements of the Pareto curve are achievable solutions.

3. The problem whether a given vector represents an achievable solution is solvable in polynomial time.

4.a For the expectation objectives, a strategy which achieves a given solution is computable in polynomial time.

4.b For the satisfaction objectives, a strategy which $\varepsilon$-approximates a given solution is computable in polynomial time.

5. The problem whether a given vector belongs to the Pareto curve is solvable in polynomial time.

6. A finite description of the Pareto curve is computable in exponential time. Further, an $\varepsilon$-approximate Pareto curve is computable in time which is polynomial in $1 / \varepsilon$, the size of a given MDP and the maximal absolute value of a reward assigned, and exponential in the number of mean-payoff functions.

A more detailed and precise explanation of our results is postponed to Section 3 
Let us note that MDPs with multiple mean-payoff functions under the expectation objective were also studied in [7], and it was claimed that memoryless randomized strategies are sufficient for $\varepsilon$-approximation of the Pareto curve, for all $\varepsilon>0$, and an NP algorithm was presented to find a memoryless randomized strategy achieving a given vector. We show with an example that under the expectation objective there exists $\varepsilon>0$ such that randomized strategies do require memory for $\varepsilon$-approximation, and thus reveal a flaw in the earlier paper.

Similarly to the related papers [8, 10, 12] (see Related Work), we obtain our results by a characterization of the set of achievable solutions by a set of linear constraints, and from the linear constraints we construct witness strategies for any achievable solution. However, our approach differs significantly from the previous work. In all the previous works, the linear constraints are used to encode a memoryless strategy either directly for the MDP [8], or (if memoryless strategies do not suffice in general) for a finite "product" of the MDP and the specification function expressed as automata, from which the memoryless strategy is then transferred to a finite-memory strategy for the original MDP [10, 12, 9]. In our setting new problems arise. Under the expectation objective with mean-payoff function, neither is there any immediate notion of "product" of MDP and mean-payoff function and nor do memoryless strategies suffice. Moreover, even for memoryless strategies the linear constraint characterization is not straightforward for mean-payoff functions, as in the case of discounted [8], reachability [10] and total reward functions [12]: for example, in [7] even for memoryless strategies there was no linear constraint characterization for mean-payoff function and only an NP algorithm was given. Our result, obtained by a characterization of linear constraints directly on the original MDP, requires involved and intricate construction of witness strategies. Moreover, our results are significant and non-trivial generalizations of the classical results for MDPs with a single mean-payoff function, where memoryless pure optimal strategies exist, while for multiple functions both randomization and memory is necessary. Under the satisfaction objective, any finite product on which a memoryless strategy would exist is not feasible as in general witness strategies for achievable solutions may need an infinite amount of memory. We establish a correspondence between the set of achievable solutions under both types of objectives for strongly connected MDPs. Finally, we use this correspondence to obtain our result for satisfaction objectives.

A conference version of this work was published at the conference LICS 2011 [3].

Related Work. The study of Markov decision processes with multiple expectation objectives has been initiated in the area of applied probability theory, where it is known as constrained MDPs [18, 1]. The attention in the study of constrained MDPs has been focused mainly to restricted classes of MDPs, such as unichain MDPs where all states are visited infinitely often under any strategy. Such restriction both guarantees the existence of memoryless optimal strategies as well as simpler linear programming based algorithm for the problem, than the general case studied in this paper.

For general finite-state MDPs, [8] studied MDPs with multiple discounted reward functions. It was shown that memoryless strategies suffice for Pareto optimization, and a polynomial-time algorithm was given to approximate (up to a given relative error) the Pareto curve by reduction to multi-objective linear programming and using the results of [17]. MDPs with multiple qualitative $\omega$-regular specifications were studied in [10]. It was shown that the Pareto curve can be approximated in polynomial time; the algorithm reduces the problem to MDPs with multiple reachability specifications, which can be solved by multi-objective linear programming. In [12], the results of [10] were extended to combine 
$\omega$-regular and expected total reward objectives. MDPs with multiple mean-payoff functions under expectation objectives were considered in [7, and our analysis reveals flaws in the earlier paper, correct the flaws, and allows us to present significantly improved results (a polynomial-time algorithm for finding a strategy achieving a given vector as compared to the previously suggested incorrect NP algorithm). Moreover, the satisfaction objective has not been considered in multi-objective setting before, and even in single objective case it has been considered only in a very specific setting [4].

\section{Preliminaries}

We use $\mathbb{N}, \mathbb{Z}, \mathbb{Q}$, and $\mathbb{R}$ to denote the sets of positive integers, integers, rational numbers, and real numbers, respectively. Given two vectors $\vec{v}, \vec{u} \in \mathbb{R}^{k}$, where $k \in \mathbb{N}$, we write $\vec{v} \leq \vec{u}$ iff $\vec{v}_{i} \leq \vec{u}_{i}$ for all $1 \leq i \leq k$, and $\vec{v}<\vec{u}$ iff $\vec{v} \leq \vec{u}$ and $\vec{v}_{i}<\vec{u}_{i}$ for some $1 \leq i \leq k$.

We assume familiarity with basic notions of probability theory, e.g., probability space, random variable, or expected value. As usual, a probability distribution over a finite or countably infinite set $X$ is a function $f: X \rightarrow[0,1]$ such that $\sum_{x \in X} f(x)=1$. We call $f$ positive if $f(x)>0$ for every $x \in X$, rational if $f(x) \in \mathbb{Q}$ for every $x \in X$, and Dirac if $f(x)=1$ for some $x \in X$. The set of all distributions over $X$ is denoted by $\operatorname{dist}(X)$.

Markov chains. A Markov chain is a tuple $M=(L, \rightarrow, \mu)$ where $L$ is a finite or countably infinite set of locations, $\rightarrow \subseteq L \times(0,1] \times L$ is a transition relation such that for each fixed $\ell \in L, \sum_{\ell \rightarrow \ell^{\prime}} x=1$, and $\mu$ is the initial probability distribution on $L$.

A run in $M$ is an infinite sequence $\omega=\ell_{1} \ell_{2} \ldots$ of locations such that $\ell_{i} \stackrel{x}{\rightarrow} \ell_{i+1}$ for every $i \in \mathbb{N}$. A finite path in $M$ is a finite prefix of a run. Each finite path $w$ in $M$ determines the set Cone $(w)$ consisting of all runs that start with $w$. To $M$ we associate the probability space $\left(\operatorname{Runs}_{M}, \mathcal{F}, \mathbb{P}\right)$, where Runs $M$ is the set of all runs in $M, \mathcal{F}$ is the $\sigma$-field generated by all Cone $(w)$, and $\mathbb{P}$ is the unique probability measure such that $\mathbb{P}\left(\operatorname{Cone}\left(\ell_{1}, \ldots, \ell_{k}\right)\right)=$ $\mu\left(\ell_{1}\right) \cdot \prod_{i=1}^{k-1} x_{i}$, where $\ell_{i} \stackrel{x_{i}}{\rightarrow} \ell_{i+1}$ for all $1 \leq i<k$ (the empty product is equal to 1 ).

Markov decision processes. A Markov decision process (MDP) is a tuple of the form $G=(S, A, A c t, \delta)$ where $S$ is a finite set of states, $A$ is a finite set of actions, Act $: S \rightarrow$ $2^{A} \backslash\{\emptyset\}$ is an action enabledness function that assigns to each state $s$ the set $\operatorname{Act}(s)$ of actions enabled at $s$, and $\delta: S \times A \rightarrow \operatorname{dist}(S)$ is a probabilistic transition function that given a state $s$ and an action $a \in A c t(s)$ enabled at $s$ gives a probability distribution over the successor states. For simplicity, we assume that every action is enabled in exactly one state, and we denote this state $\operatorname{Src}(a)$. Thus, henceforth we will assume that $\delta: A \rightarrow \operatorname{dist}(S)$.

A run in $G$ is an infinite alternating sequence of states and actions $\omega=s_{1} a_{1} s_{2} a_{2} \ldots$ such that for all $i \geq 1, \operatorname{Src}\left(a_{i}\right)=s_{i}$ and $\delta\left(a_{i}\right)\left(s_{i+1}\right)>0$. We denote by Runs $G$ the set of all runs in $G$. A finite path of length $k$ in $G$ is a finite prefix $w=s_{1} a_{1} \ldots a_{k-1} s_{k}$ of a run in $G$. For a finite path $w$ we denote by last $(w)$ the last state of $w$.

A pair $(T, B)$ with $\emptyset \neq T \subseteq S$ and $B \subseteq \bigcup_{t \in T} A c t(t)$ is an end component of $G$ if (1) for all $a \in B$, whenever $\delta(a)\left(s^{\prime}\right)>0$ then $s^{\prime} \in T$; and (2) for all $s, t \in T$ there is a finite path $\omega=s_{1} a_{1} \ldots a_{k-1} s_{k}$ such that $s_{1}=s, s_{k}=t$, and all states and actions that appear in $\omega$ belong to $T$ and $B$, respectively. An end component $(T, B)$ is a maximal end component $(M E C)$ if it is maximal wrt. pointwise subset ordering. Given an end component $C=(T, B)$, we sometimes abuse notation by using $C$ instead of $T$ or $B$, e.g., by writing $a \in C$ instead of $a \in B$ for $a \in A$. 
Strategies and plays. Intuitively, a strategy in an MDP $G$ is a "recipe" to choose actions. Usually, a strategy is formally defined as a function $\sigma:(S A)^{*} S \rightarrow \operatorname{dist}(A)$ that given a finite path $w$, representing the history of a play, gives a probability distribution over the actions enabled in last $(w)$. In this paper, we adopt a somewhat different (though equivalent - see Section (6) definition, which allows a more natural classification of various strategy types. Let $\mathrm{M}$ be a finite or countably infinite set of memory elements. A strategy is a triple $\sigma=\left(\sigma_{u}, \sigma_{n}, \alpha\right)$, where $\sigma_{u}: A \times S \times \mathrm{M} \rightarrow \operatorname{dist}(\mathrm{M})$ and $\sigma_{n}: S \times \mathrm{M} \rightarrow \operatorname{dist}(A)$ are memory update and next move functions, respectively, and $\alpha$ is an initial distribution on memory elements. We require that for all $(s, m) \in S \times \mathrm{M}$, the distribution $\sigma_{n}(s, m)$ assigns a positive value only to actions enabled at $s$. The set of all strategies is denoted by $\Sigma$ (the underlying MDP $G$ will be always clear from the context).

Let $s \in S$ be an initial state. A play of $G$ determined by $s$ and a strategy $\sigma$ is a Markov chain $G_{s}^{\sigma}$ (or just $G^{\sigma}$ if $s$ is clear from the context) where the set of locations is $S \times \mathrm{M} \times A$, the initial distribution $\mu$ is positive only on (some) elements of $\{s\} \times \mathrm{M} \times A$ where $\mu(s, m, a)=\alpha(m) \cdot \sigma_{n}(s, m)(a)$, and $(t, m, a) \stackrel{x}{\rightarrow}\left(t^{\prime}, m^{\prime}, a^{\prime}\right)$ iff

$$
x=\delta(a)\left(t^{\prime}\right) \cdot \sigma_{u}\left(a, t^{\prime}, m\right)\left(m^{\prime}\right) \cdot \sigma_{n}\left(t^{\prime}, m^{\prime}\right)\left(a^{\prime}\right)>0 .
$$

Hence, $G_{s}^{\sigma}$ starts in a location chosen randomly according to $\alpha$ and $\sigma_{n}$. In a current location $(t, m, a)$, the next action to be performed is $a$, hence the probability of entering $t^{\prime}$ is $\delta(a)\left(t^{\prime}\right)$. The probability of updating the memory to $m^{\prime}$ is $\sigma_{u}\left(a, t^{\prime}, m\right)\left(m^{\prime}\right)$, and the probability of selecting $a^{\prime}$ as the next action is $\sigma_{n}\left(t^{\prime}, m^{\prime}\right)\left(a^{\prime}\right)$. We assume that these choices are independent, and thus obtain the product above.

In this paper, we consider various functions over Runs $G$ that become random variables over Runs $_{G_{s}^{\sigma}}$ after fixing some $\sigma$ and $s$. For example, for $F \subseteq S$ we denote by $\operatorname{Reach}(F) \subseteq$ $\operatorname{Runs}_{G}$ the set of all runs reaching $F$. Then $\operatorname{Reach}(F)$ naturally determines $\operatorname{Reach}_{s}^{\sigma}(F) \subseteq$ Runs $_{G_{s}^{\sigma}}$ by simply "ignoring" the visited memory elements. To simplify and unify our notation, we write, e.g., $\mathbb{P}_{s}^{\sigma}[\operatorname{Reach}(F)]$ instead of $\mathbb{P}_{s}^{\sigma}\left[\operatorname{Reach} s_{s}^{\sigma}(F)\right]$, where $\mathbb{P}_{s}^{\sigma}$ is the probability measure of the probability space associated to $G_{s}^{\sigma}$. We also adopt this notation for other events and functions, such as $\operatorname{lr}_{\text {inf }}(\vec{r})$ or $\operatorname{lr}_{\text {sup }}(\vec{r})$ defined in the next section, and write, e.g., $\mathbb{E}_{s}^{\sigma}\left[\operatorname{lr}_{\text {inf }}(\vec{r})\right]$ instead of $\mathbb{E}\left[\operatorname{lr}_{\text {inf }}(\vec{r})_{s}^{\sigma}\right]$.

Strategy types. In general, a strategy may use infinite memory, and both $\sigma_{u}$ and $\sigma_{n}$ may randomize. According to the use of randomization, a strategy, $\sigma$, can be classified as

- pure (or deterministic), if $\alpha$ is Dirac and both the memory update and the next move function give a Dirac distribution for every argument;

- deterministic-update, if $\alpha$ is Dirac and the memory update function gives a Dirac distribution for every argument;

- stochastic-update, if $\alpha, \sigma_{u}$, and $\sigma_{n}$ are unrestricted.

Note that every pure strategy is deterministic-update, and every deterministic-update strategy is stochastic-update. A randomized strategy is a strategy which is not necessarily pure. We also classify the strategies according to the size of memory they use. Important subclasses are memoryless strategies, in which $\mathrm{M}$ is a singleton, $n$-memory strategies, in which $\mathrm{M}$ has exactly $n$ elements, and finite-memory strategies, in which $\mathrm{M}$ is finite. By $\Sigma^{M}$ we denote the set of all memoryless strategies. Memoryless strategies can be specified as $\sigma: S \rightarrow \operatorname{dist}(A)$. Memoryless pure strategies, i.e., those which are both pure and memoryless, can be specified as $\sigma: S \rightarrow A$. 

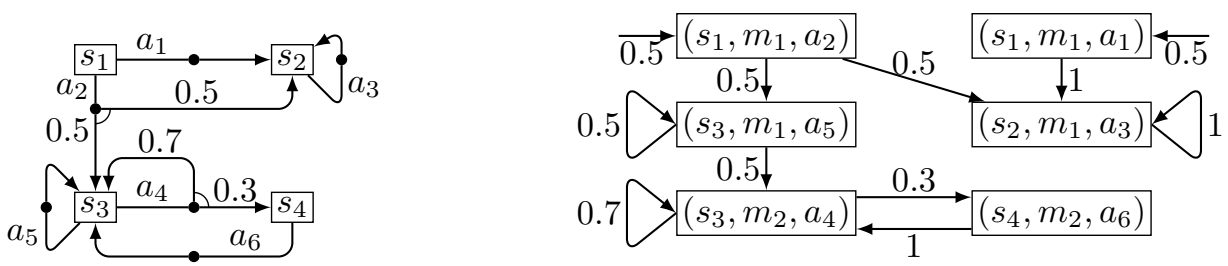

FiguRE 1. Running example MDP (left) and its play (right)

For a finite-memory strategy $\sigma$, a bottom strongly connected component (BSCC) of $G_{s}^{\sigma}$ is a subset of locations $W \subseteq S \times \mathrm{M} \times A$ such that for all $\ell_{1} \in W$ and $\ell_{2} \in S \times \mathrm{M} \times A$ we have that (i) if $\ell_{2}$ is reachable from $\ell_{1}$, then $\ell_{2} \in W$, and (ii) for all $\ell_{1}, \ell_{2} \in W$ we have that $\ell_{2}$ is reachable from $\ell_{1}$. Every BSCC $W$ determines a unique end component $(\{s \mid(s, m, a) \in W\},\{a \mid(s, m, a) \in W\})$ of $G$, and we sometimes do not strictly distinguish between $W$ and its associated end component.

As we already noted, stochastic-update strategies can be easily translated into "ordinary" strategies of the form $\sigma:(S A)^{*} S \rightarrow \operatorname{dist}(A)$, and vice versa (see Section 6). Note that a finite-memory stochastic-update strategy $\sigma$ can be easily implemented by a stochastic finite-state automaton that scans the history of a play "on the fly" (in fact, $G_{s}^{\sigma}$ simulates this automaton). Hence, finite-memory stochastic-update strategies can be seen as natural extensions of ordinary (i.e., deterministic-update) finite-memory strategies that are implemented by deterministic finite-state automata.

A running example (I). As an example, consider the $\mathrm{MDP} G=(S, A, A c t, \delta)$ of Figure 1 (left). Here, $S=\left\{s_{1}, \ldots, s_{4}\right\}, A=\left\{a_{1}, \ldots, a_{6}\right\}$, Act is denoted using the labels on lines going from actions, e.g., $\operatorname{Act}\left(s_{1}\right)=\left\{a_{1}, a_{2}\right\}$, and $\delta$ is given by the arrows, e.g., $\delta\left(a_{4}\right)\left(s_{4}\right)=0.3$. Note that $G$ has four end components (one on $\left\{s_{2}\right\}$, another on $\left\{s_{3}\right\}$, and two on $\left.\left\{s_{3}, s_{4}\right\}\right)$ and two MECs.

Let $s_{1}$ be the initial state and $\mathrm{M}=\left\{m_{1}, m_{2}\right\}$. Consider a stochastic-update finitememory strategy $\sigma=\left(\sigma_{u}, \sigma_{n}, \alpha\right)$ where $\alpha$ chooses $m_{1}$ deterministically, and $\sigma_{n}\left(m_{1}, s_{1}\right)=$ $\left[a_{1} \mapsto 0.5, a_{2} \mapsto 0.5\right], \sigma_{n}\left(m_{2}, s_{3}\right)=\left[a_{4} \mapsto 1\right]$ and otherwise $\sigma_{n}$ chooses self-loops. The memory update function $\sigma_{u}$ leaves the memory intact except for the case $\sigma_{u}\left(m_{1}, s_{3}\right)$ where both $m_{1}$ and $m_{2}$ are chosen with probability 0.5. The play $G_{s_{1}}^{\sigma}$ is depicted in Figure 1 (right).

\section{Main Results}

In this paper we establish basic results about Markov decision processes with expectation and satisfaction objectives specified by multiple limit-average (or mean-payoff) functions. We adopt the variant where rewards are assigned to edges (i.e., actions) rather than states of a given MDP.

Let $G=(S, A, A c t, \delta)$ be a MDP, and $r: A \rightarrow \mathbb{Q}$ a reward function. Note that $r$ may also take negative values. For every $j \in \mathbb{N}$, let $A_{j}:$ Runs $_{G} \rightarrow A$ be a function which to every run $\omega \in$ Runs $_{G}$ assigns the $j$-th action of $\omega$. Since the limit-average function $\operatorname{lr}(r):$ Runs $_{G} \rightarrow \mathbb{R}$ given by

$$
\operatorname{lr}(r)(\omega)=\lim _{T \rightarrow \infty} \frac{1}{T} \sum_{t=1}^{T} r\left(A_{t}(\omega)\right)
$$


may be undefined for some runs, we consider its lower and upper approximation $\operatorname{lr}_{\text {inf }}(r)$ and $\operatorname{lr}_{\text {sup }}(r)$ that are defined for all $\omega \in$ Runs as follows:

$$
\begin{aligned}
\operatorname{lr}_{\text {inf }}(r)(\omega) & =\liminf _{T \rightarrow \infty} \frac{1}{T} \sum_{t=1}^{T} r\left(A_{t}(\omega)\right), \\
\operatorname{lr}_{\text {sup }}(r)(\omega) & =\limsup _{T \rightarrow \infty} \frac{1}{T} \sum_{t=1}^{T} r\left(A_{t}(\omega)\right) .
\end{aligned}
$$

For a vector $\vec{r}=\left(r_{1}, \ldots, r_{k}\right)$ of reward functions, we similarly define the $\mathbb{R}^{k}$-valued functions

$$
\begin{aligned}
\operatorname{lr}(\vec{r}) & =\left(\operatorname{lr}\left(r_{1}\right), \ldots, \operatorname{lr}\left(r_{k}\right)\right), \\
\operatorname{lr}_{\text {inf }}(\vec{r}) & =\left(\operatorname{lr}_{\mathrm{inf}}\left(r_{1}\right), \ldots, \operatorname{lr}_{\mathrm{inf}}\left(r_{k}\right)\right), \\
\operatorname{lr}_{\mathrm{sup}}(\vec{r}) & =\left(\operatorname{lr}_{\mathrm{sup}}\left(r_{1}\right), \ldots, \operatorname{lr}_{\mathrm{sup}}\left(r_{k}\right)\right) .
\end{aligned}
$$

We sometimes refer to "runs satisfying $\operatorname{lr}(\vec{r}) \geq \vec{v}$ " instead of "runs $\omega$ satisfying $\operatorname{lr}(\vec{r})(\omega) \geq \vec{v}$ ".

Now we introduce the expectation and satisfaction objectives determined by $\vec{r}$.

- The expectation objective amounts to maximizing or minimizing the expected value of $\operatorname{lr}(\vec{r})$. Since $\operatorname{lr}(\vec{r})$ may be undefined for some runs, we actually aim at maximizing the expected value of $\operatorname{lr}_{\text {inf }}(\vec{r})$ or minimizing the expected value of $\operatorname{lr}_{\text {sup }}(\vec{r})$ (wrt. componentwise ordering $\leq$ ).

- The satisfaction objective means maximizing the probability of all runs where $\operatorname{lr}(\vec{r})$ stays above or below a given vector $\vec{v}$. Technically, we aim at maximizing the probability of all runs where $\operatorname{lr}_{\inf }(\vec{r}) \geq \vec{v}$, or at maximizing the probability of all runs where $\operatorname{lr}_{\text {sup }}(\vec{r}) \leq \vec{v}$.

The expectation objective is relevant in situations when we are interested in the average or aggregate behaviour of many instances of a system, and in contrast, the satisfaction objective is relevant when we are interested in particular executions of a system and wish to optimize the probability of generating the desired executions. $\operatorname{Since} \operatorname{lr}_{\mathrm{inf}}(\vec{r})=-\operatorname{lr}_{\mathrm{sup}}(-\vec{r})$, the problems of maximizing and minimizing the expected value of $\operatorname{lr}_{\text {inf }}(\vec{r})$ and $\operatorname{lr}_{\text {sup }}(\vec{r})$ are dual. Therefore, we consider just the problem of maximizing the expected value of $\operatorname{lr}_{\text {inf }}(\vec{r})$. For the same reason, we consider only the problem of maximizing the probability of all runs where $\operatorname{lr}_{\text {inf }}(\vec{r}) \geq \vec{v}$.

If $k$ (the dimension of $\vec{r}$ ) is at least two, there might be several incomparable solutions to the expectation objective; and if $\vec{v}$ is slightly changed, the achievable probability of all runs satisfying $\operatorname{lr}_{\text {inf }}(\vec{r}) \geq \vec{v}$ may change considerably. Therefore, we aim not only at constructing a particular solution, but on characterizing and approximating the whole space of achievable solutions for the expectation/satisfaction objective. Let $s \in S$ be some (initial) state of $G$. We define the sets $\operatorname{AcEx}\left(\operatorname{lr}_{\inf }(\vec{r})\right)$ and $\operatorname{AcSt}\left(\operatorname{lr}_{\text {inf }}(\vec{r})\right)$ of achievable vectors for the expectation and satisfaction objectives as follows:

$$
\begin{aligned}
\operatorname{AcEx}\left(\operatorname{lr}_{\text {inf }}(\vec{r})\right) & =\left\{\vec{v} \mid \exists \sigma \in \Sigma: \mathbb{E}_{s}^{\sigma}\left[\operatorname{lr}_{\text {inf }}(\vec{r})\right] \geq \vec{v}\right\}, \\
\operatorname{AcSt}\left(\operatorname{lr}_{\inf }(\vec{r})\right) & =\left\{(\nu, \vec{v}) \mid \exists \sigma \in \Sigma: \mathbb{P}_{s}^{\sigma}\left[\operatorname{lr}_{\text {inf }}(\vec{r}) \geq \vec{v}\right] \geq \nu\right\} .
\end{aligned}
$$

Intuitively, if $\vec{v}, \vec{u}$ are achievable vectors such that $\vec{v}>\vec{u}$, then $\vec{v}$ represents a "strictly better" solution than $\vec{u}$. The set of "optimal" solutions defines the Pareto curve for $\operatorname{AcEx}\left(\operatorname{lr}_{\inf }(\vec{r})\right)$ and $\operatorname{AcSt}\left(\operatorname{lr}_{\text {inf }}(\vec{r})\right)$. In general, the Pareto curve for a given set $Q \subseteq \mathbb{R}^{k}$ is the set $P$ of all minimal vectors $\vec{v} \in \mathbb{R}^{k}$ such $\vec{v} \nless \vec{u}$ for all $\vec{u} \in Q$. Note that $P$ may contain vectors that are not in $Q$ (for example, if $Q=\{x \in \mathbb{R} \mid x<2\}$, then $P=\{2\}$ ). However, every vector $\vec{v} \in P$ is "almost" in $Q$ in the sense that for every $\varepsilon>0$ there is $\vec{u} \in Q$ with $\vec{v} \leq \vec{u}+\vec{\varepsilon}$, 


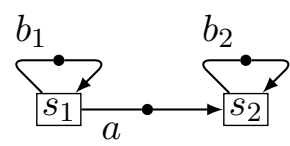

FiguRE 2. Example of insufficiency of memoryless strategies

where $\vec{\varepsilon}=(\varepsilon, \ldots, \varepsilon)$. This naturally leads to the notion of an $\varepsilon$-approximate Pareto curve, $P_{\varepsilon}$, which is a subset of $Q$ such that for all vectors $\vec{v} \in P$ of the Pareto curve there is a vector $\vec{u} \in P_{\varepsilon}$ such that $\vec{v} \leq \vec{u}+\vec{\varepsilon}$. Note that $P_{\varepsilon}$ is not unique.

A running example (II). Consider again the MDP $G$ of Figure 1 (left), and the strategy $\sigma$ constructed in our running example (I). Let $\vec{r}=\left(r_{1}, r_{2}\right)$, where $r_{1}\left(a_{6}\right)=1, r_{2}\left(a_{3}\right)=2$, $r_{2}\left(a_{4}\right)=1$, and otherwise the rewards are zero. Let

$$
\omega=\left(s 1, m 1, a_{2}\right)\left(s_{3}, m_{1}, a_{5}\right)\left(\left(s_{3}, m_{2}, a_{4}\right)\left(s_{4}, m_{2}, a_{6}\right)\right)^{\omega}
$$

Then $\operatorname{lr}(\vec{r})(\omega)=(0.5,0.5)$. Considering the expectation objective, we have that $\mathbb{E}_{s_{1}}^{\sigma}\left[\operatorname{lr}_{\text {inf }}(\vec{r})\right]=$ $\left(\frac{3}{52}, \frac{22}{13}\right)$. Considering the satisfaction objective, we have that $(0.5,0,2) \in \operatorname{AcSt}(\vec{r})$ because $\mathbb{P}_{s_{1}}^{\sigma}\left[\operatorname{lr}_{\text {inf }}(\vec{r}) \geq(0,2)\right]=0.5$. The Pareto curve for $\operatorname{AcEx}\left(\operatorname{lr}_{\text {inf }}(\vec{r})\right)$ consists of the points $\left\{\left(\frac{3}{13} x, \frac{10}{13} x+2(1-x)\right) \mid 0 \leq x \leq 0.5\right\}$, and the Pareto curve for $\operatorname{AcSt}\left(\operatorname{lr}_{\inf }(\vec{r})\right)$ is $\{(1,0,2)\} \cup$ $\left\{(0.5, x, 1-x) \mid 0<x_{1} \leq \frac{10}{13}\right\}$.

Now we are equipped with all the notions needed for understanding the main results of this paper. Our work is motivated by the six fundamental questions given in Section 1. In the next subsections we give detailed answers to these questions.

3.1. Expectation objectives. The answers to Q.1-Q.6 for the expectation objectives are the following:

A.1 For all achievable solutions, 2-memory stochastic-update strategies are sufficient, i.e., for all $\vec{v} \in \operatorname{AcEx}\left(\operatorname{lr}_{\mathrm{inf}}(\vec{r})\right)$ there is a 2-memory stochastic-update strategy $\sigma$ satisfying $\mathbb{E}_{s}^{\sigma}\left[\operatorname{lr}_{\text {inf }}(\vec{r})\right] \geq \vec{v}$.

A.2 The Pareto curve $P$ for $\operatorname{AcEx}\left(\operatorname{lr}_{\text {inf }}(\vec{r})\right)$ is a subset of $\operatorname{AcEx}\left(\operatorname{lr}_{\text {inf }}(\vec{r})\right)$, i.e., all optimal solutions are achievable.

A.3 There is a polynomial-time algorithm which, given any $\vec{v} \in \mathbb{Q}^{k}$, decides whether $\vec{v} \in$ $\operatorname{AcEx}\left(\operatorname{lr}_{\inf }(\vec{r})\right)$.

A.4 If $\vec{v} \in \operatorname{AcEx}\left(\operatorname{lr}_{\text {inf }}(\vec{r})\right)$, then there is a 2-memory stochastic-update strategy $\sigma$ constructible in polynomial time satisfying $\mathbb{E}_{s}^{\sigma}\left[\operatorname{lr}_{\text {inf }}(\vec{r})\right] \geq \vec{v}$.

A.5 There is a polynomial-time algorithm which, given $\vec{v} \in \mathbb{R}^{k}$, decides whether $\vec{v}$ belongs to the Pareto curve for $\operatorname{AcEx}\left(\operatorname{lr}_{\text {inf }}(\vec{r})\right)$.

A.6 There is a convex hull $Z$ of finitely many vectors such that: $\operatorname{AcEx}\left(\operatorname{lr}_{\mathrm{inf}}(\vec{r})\right)$ is a downward closure of $Z$ (i.e. $\operatorname{AcEx}(\operatorname{lr} \inf (\vec{r}))=\{\vec{v} \mid \exists \vec{u} \in Z: \vec{v} \leq \vec{u}\})$; The Pareto curve for $\operatorname{AcEx}\left(\operatorname{lr}_{\mathrm{inf}}(\vec{r})\right)$ is a union of all facets of $Z$ whose vectors are not strictly dominated by vectors of $Z$. Further, an $\varepsilon$-approximate Pareto curve for $\operatorname{AcEx}\left(\operatorname{lr}_{\text {inf }}(\vec{r})\right)$ is computable in time polynomial in $\frac{1}{\varepsilon},|G|$, and $\max _{a \in A} \max _{1 \leq i \leq k}\left|\vec{r}_{i}(a)\right|$, and exponential in $k$.

Let us note that A.1 is tight in the sense that neither memoryless randomized nor pure strategies are sufficient for achievable solutions. This is witnessed by the MDP of Figure 2 with reward functions $r_{1}, r_{2}$ such that $r_{i}\left(b_{i}\right)=1$ and $r_{i}\left(b_{j}\right)=0$ for $i \neq j$. Consider a strategy $\sigma$ which initially selects between the actions $b_{1}$ and $a$ randomly (with probability 0.5 ) and 
then keeps selecting $b_{1}$ or $b_{2}$, whichever is available. Hence, $\mathbb{E}_{s_{1}}^{\sigma}\left[\operatorname{lr}_{\text {inf }}\left(\left(r_{1}, r_{2}\right)\right)\right]=(0.5,0.5)$. However, the vector $(0.5,0.5)$ is not achievable by a strategy $\sigma^{\prime}$ which is memoryless or pure, because then we inevitably have that $\mathbb{E}_{s_{1}}^{\sigma^{\prime}}\left[\operatorname{lr}_{\mathrm{inf}}\left(\left(r_{1}, r_{2}\right)\right)\right]$ is equal either to $(0,1)$ or $(1,0)$. The example also shows that memory and randomization is needed for $\varepsilon$-approximation. Considering e.g. $\varepsilon=0.1$, a history-dependent randomized strategy is needed to achieve the value $(0.5-0.1,0.5-0.1)$ or better.

The 2-memory stochastic-update strategy from A.1 and A.4 operates in two modes. Starting in the first mode, it reaches the MECs of the MDP with appropriate probabilities; once a MEC is reached, the strategy stochastically switches to a second mode, never leaving the current MEC and ensuring certain "frequencies" of taking the actions of the MEC. Since both modes can be implemented by memoryless strategies, we get that we only require two memory elements to remember which mode is currently being executed. We also show that the 2-memory stochastic-update strategy constructed can be efficiently transformed into a finite-memory deterministic-update randomized strategy, and hence the answers A.1 and A.4 are also valid for finite-memory deterministic-update randomized strategies (see Section 4.1). Observe that A.2 can be seen as a generalization of the well-known result for single payoff functions which says that finite-state MDPs with mean-payoff objectives have optimal strategies (in this case, the Pareto curve consists of a single number known as the "value"). Also observe that A.2 does not hold for infinite-state MDPs (a counterexample is simple to construct even for a single reachability objective, see e.g. [5, Example 6]).

Finally, note that if $\sigma$ is a finite-memory stochastic-update strategy, then $G_{s}^{\sigma}$ is a finitestate Markov chain. Hence, for almost all runs $\omega$ in $G_{s}^{\sigma}$ we have that $\operatorname{lr}(\vec{r})(\omega)$ exists and it is equal to $\operatorname{lr}_{\text {inf }}(\vec{r})(\omega)$. This means that there is actually no difference between maximizing the expected value of $\operatorname{lr}_{\text {inf }}(\vec{r})$ and maximizing the expected value of $\operatorname{lr}(\vec{r})$ over all strategies for which $\operatorname{lr}(\vec{r})$ exists.

3.2. Satisfaction objectives. The answers to Q.1-Q.6 for the satisfaction objectives are presented below.

B.1 Achievable vectors require strategies with infinite memory in general. However, memoryless randomized strategies are sufficient for $\varepsilon$-approximate achievable vectors; in fact, a stronger claim holds and for every $\varepsilon>0$ and $(\nu, \vec{v}) \in \operatorname{AcSt}\left(\operatorname{lr}_{\text {inf }}(\vec{r})\right)$, there is a memoryless randomized strategy $\sigma$ with

$$
\mathbb{P}_{s}^{\sigma}\left[\operatorname{lr}_{\text {inf }}(\vec{r}) \geq \vec{v}-\vec{\varepsilon}\right] \geq \nu
$$

Here $\vec{\varepsilon}=(\varepsilon, \ldots, \varepsilon)$.

B.2 The Pareto curve $P$ for $\operatorname{AcSt}\left(\operatorname{lr}_{\text {inf }}(\vec{r})\right)$ is a subset of $\operatorname{AcSt}\left(\operatorname{lr} r_{\text {inf }}(\vec{r})\right)$, i.e., all optimal solutions are achievable.

B.3 There is a polynomial-time algorithm which, given $\nu \in[0,1]$ and $\vec{v} \in \mathbb{Q}^{k}$, decides whether $(\nu, \vec{v}) \in \operatorname{AcSt}\left(\operatorname{lr}_{\inf }(\vec{r})\right)$.

B.4 If $(\nu, \vec{v}) \in \operatorname{AcSt}\left(\operatorname{lr}_{\inf }(\vec{r})\right)$, then for every $\varepsilon>0$ there is a memoryless randomized strategy $\sigma$ constructible in polynomial time such that $\mathbb{P}_{s}^{\sigma}\left[\operatorname{lr}_{\text {inf }}(\vec{r}) \geq \vec{v}-\vec{\varepsilon}\right] \geq \nu-\varepsilon$.

B.5 There is a polynomial-time algorithm which, given $\nu \in[0,1]$ and $\vec{v} \in \mathbb{R}^{k}$, decides whether $(\nu, \vec{v})$ belongs to the Pareto curve for $\operatorname{AcSt}\left(\operatorname{lr}_{\text {inf }}(\vec{r})\right)$.

B.6 The Pareto curve $P$ for $\operatorname{AcSt}\left(\ln _{\text {inf }}(\vec{r})\right)$ may be neither connected, nor closed. However, $P$ is a union of finitely many sets whose closures are convex polytopes, and, perhaps surprisingly, the set $\{\nu \mid(\nu, \vec{v}) \in P\}$ is always finite. The sets in the union that 


$$
\begin{array}{rlr}
\mathbf{1}_{s_{0}}(s)+\sum_{a \in A} y_{a} \cdot \delta(a)(s) & =\sum_{a \in A c t(s)} y_{a}+y_{s} & \\
\sum_{s \in S_{M E C}} y_{s} & =1 & \text { for all } s \in S \\
\sum_{s \in C} y_{s} & =\sum_{a \in A \cap C} x_{a} & \\
\sum_{a \in A} x_{a} \cdot \delta(a)(s) & =\sum_{a \in A c t(s)} x_{a} & \text { for all MEC } C \text { of } G \\
\sum_{a \in A} x_{a} \cdot \vec{r}_{i}(a) & \geq \vec{v}_{i} & \text { for all } s \in S
\end{array}
$$

FiguRE 3. System $L$ of linear inequalities for Theorem 4.1. (We define $S_{M E C} \subseteq S$ to be the states contained in some MEC of $G, \mathbf{1}_{s_{0}}(s)=1$ if $s=s_{0}$, and $\mathbf{1}_{s_{0}}(s)=0$ otherwise.)

gives $P$ (resp. the inequalities that define them) can be computed. Further, an $\varepsilon$ approximate Pareto curve for $\operatorname{AcSt}\left(\operatorname{lr}_{\text {inf }}(\vec{r})\right)$ is computable in time polynomial in $\frac{1}{\varepsilon},|G|$, and $\max _{a \in A} \max _{1 \leq i \leq k}\left|\vec{r}_{i}(a)\right|$, and exponential in $k$.

The algorithms of B.3 and B.4 are polynomial in the size of $G$ and the size of binary representations of $\vec{v}$ and $\frac{1}{\varepsilon}$.

The result B.1 is again tight. In Lemma 5.2 we show that memoryless pure strategies are insufficient for $\varepsilon$-approximate achievable vectors, i.e., there are $\varepsilon>0$ and $(\nu, \vec{v}) \in$ $\operatorname{AcSt}\left(\operatorname{lr}_{\inf }(\vec{r})\right)$ such that for every memoryless pure strategy $\sigma$ we have $\mathbb{P}_{s}^{\sigma}\left[\operatorname{lr}_{\inf }(\vec{r}) \geq \vec{v}-\vec{\varepsilon}\right]<$ $\nu-\varepsilon$.

As noted in B.1, a strategy $\sigma$ achieving a given vector $(\nu, \vec{v}) \in \operatorname{AcSt}\left(\operatorname{lr}_{\text {inf }}(\vec{r})\right)$ may require infinite memory. Still, our proof of B.1 reveals a "recipe" for constructing such a $\sigma$ by simulating the memoryless randomized strategies $\sigma_{\varepsilon}$ which $\varepsilon$-approximate $(\nu, \vec{v})$ (intuitively, for smaller and smaller $\varepsilon$, the strategy $\sigma$ simulates $\sigma_{\varepsilon}$ longer and longer; the details are

discussed in Section 51). Hence, for almost all runs $\omega$ in $G_{s}^{\sigma}$ we again have that $\operatorname{lr}(\vec{r})(\omega)$ exists and it is equal to $\operatorname{lr}_{\text {inf }}(\vec{r})(\omega)$.

\section{Solution for Expectation Objectives}

The technical core of our results for expectation objectives is the following:

Theorem 4.1. Let $G=(S, A, A c t, \delta)$ be an $M D P, s_{0} \in S$ an initial state, $\vec{r}=\left(r_{1}, \ldots, r_{k}\right)$ a tuple of reward functions, and $\vec{v} \in \mathbb{R}^{k}$. The system of linear inequalities $L$ from Figure 3 is constructible in polynomial time and satisfies:

- every nonnegative solution of $L$ induces a 2-memory stochastic-update strategy $\sigma$ satisfying $\mathbb{E}_{s_{0}}^{\sigma}\left[\operatorname{lr}_{\text {inf }}(\vec{r})\right] \geq \vec{v}$

- if $\vec{v} \in \operatorname{AcEx}\left(\operatorname{lr}_{\mathrm{inf}}(\vec{r})\right)$, then $L$ has a nonnegative solution.

As we already noted in Section 1, the proof of Theorem 4.1 is non-trivial and it is based on novel techniques and observations. Our results about expectation objectives are corollaries to Theorem 4.1 and the arguments developed in its proof. For the rest of this section, we fix an MDP $G$, a vector of rewards, $\vec{r}=\left(r_{1}, \ldots, r_{k}\right)$, and an initial state $s_{0}$ (in 
the considered plays of $G$, the initial state is not written explicitly, unless it is different from $\left.s_{0}\right)$.

Obviously, $L$ is constructible in polynomial time. Let us briefly explain the intuition behind $L$. As mentioned earlier, a 2-memory stochastic-update strategy witnessing that $\vec{v} \in \operatorname{AcEx}\left(\operatorname{lr}_{\inf }(\vec{r})\right)$ works in two modes. In the first mode it ensures that each MEC is reached and never left with certain probability, and in the second mode actions are taken with required frequencies. In $L$, the probability of reaching a MEC $C$ is encoded as the value $\sum_{s \in C} y_{s}$, and Equations (4.1) are used to ensure that the numbers obtained are indeed realisable under some strategy. The meaning of these equations is similar as the meaning of similar equations in [10, essentially the equations encode that the expected number of times a state is entered (left-hand side of the equations) is equal to the expected number of times a state is left together with probability of switching to the second mode (right-hand side of the equations). A more formal explanation of these equations is given at the end of the proof of Proposition 4.5. The frequency of taking an action $a$ is then encoded as $x_{a}$, and realisability of the solution by some strategy is ensured using Equations (4.4). Here the meaning of the equations is that the frequency with which a state is entered must be equal to the frequency with which it is left; this is formalised in Lemma 4.3.

As both directions of Theorem 4.1 are technically involved, we prove them separately as Propositions 4.2 and 4.5 .

Proposition 4.2. Every nonnegative solution of the system $L$ of Figure 3 induces a 2memory stochastic-update strategy $\sigma$ satisfying $\mathbb{E}_{s_{0}}^{\sigma}\left[\operatorname{li}_{\mathrm{inf}}(\vec{r})\right] \geq \vec{v}$.

Proof of Proposition 4.2. First, let us consider Equations (4.4) of L. Intuitively, this equation is solved by an "invariant" distribution on actions, i.e., each solution gives frequencies of actions (up to a multiplicative constant) defined for all $a \in A, s \in S$, and $\sigma \in \Sigma$ by

$$
\operatorname{freq}(\sigma, s, a):=\lim _{T \rightarrow \infty} \frac{1}{T} \sum_{t=1}^{T} \mathbb{P}_{s}^{\sigma}\left[A_{t}=a\right],
$$

assuming that the defining limit exists (which might not be the case - cf. the proof of Proposition 4.5). We prove the following:

Lemma 4.3. Assume that assigning (nonnegative) values $\bar{x}_{a}$ to $x_{a}$ solves Equations (4.4). Then there is a memoryless strategy $\xi$ such that for every BSCCs $D$ of $G^{\xi}$, every $s \in D \cap S$, and every $a \in D \cap A$, we have that freq $(\xi, s, a)$ equals a common value freq $(\xi, D, a):=$ $\bar{x}_{a} / \sum_{a^{\prime} \in D \cap A} \bar{x}_{a^{\prime}}$.

Proof. For all $s \in S$ we set $\bar{x}_{s}=\sum_{b \in A c t(s)} \bar{x}_{b}$ and define $\xi$ by $\xi(s)(a):=\frac{\bar{x}_{a}}{\bar{x}_{s}}$ if $\bar{x}_{s}>0$, and arbitrarily otherwise. We claim that the vector of values $\bar{x}_{s}$ forms an invariant measure of $G^{\xi}$. Indeed, noting that $\sum_{a \in A c t(s)} \xi(s)(a) \cdot \delta(a)\left(s^{\prime}\right)$ is the probability of the transition 
$s \rightarrow s^{\prime}$ in $G^{\xi}:$

$$
\begin{aligned}
\sum_{s \in S} \bar{x}_{s} \cdot \sum_{a \in A c t(s)} \xi(s)(a) \cdot \delta(a)\left(s^{\prime}\right) & =\sum_{s \in S} \sum_{a \in A c t(s)} \bar{x}_{s} \cdot \frac{\bar{x}_{a}}{\bar{x}_{s}} \cdot \delta(a)\left(s^{\prime}\right) \\
& =\sum_{a \in A} \bar{x}_{a} \cdot \delta(a)\left(s^{\prime}\right) \\
& =\sum_{a \in A c t\left(s^{\prime}\right)} \bar{x}_{a} \\
& =\bar{x}_{s^{\prime}} .
\end{aligned}
$$

As a consequence, $\bar{x}_{s}>0$ iff $s$ lies in some BSCC of $G^{\xi}$. Choose some BSCC $D$, and denote by $\bar{x}_{D}$ the number $\sum_{a \in D \cap A} \bar{x}_{a}=\sum_{s \in D \cap S} \bar{x}_{s}$. Also denote by $I_{t}^{a}$ the indicator of $A_{t}=a$, given by $I_{t}^{a}=1$ if $A_{t}=a$ and 0 otherwise. By the Ergodic theorem for finite Markov chains (see, e.g. [15, Theorem 1.10.2]), for all $s \in D \cap S$ and $a \in D \cap A$ we have

$$
\mathbb{E}_{s}^{\xi}\left[\lim _{T \rightarrow \infty} \frac{1}{T} \sum_{t=1}^{T} I_{t}^{a}\right]=\sum_{s^{\prime} \in D \cap S} \frac{\bar{x}_{s^{\prime}}}{\bar{x}_{D}} \cdot \xi\left(s^{\prime}\right)(a)=\frac{\bar{x}_{s^{\prime}}}{\bar{x}_{D}} \cdot \frac{\bar{x}_{a}}{\bar{x}_{s^{\prime}}}=\frac{\bar{x}_{a}}{\bar{x}_{D}} .
$$

Because $\left|I_{t}^{a}\right| \leq 1$, Lebesgue Dominated convergence theorem (see, e.g. 19, Chapter 4, Section 4]) yields $\mathbb{E}_{s}^{\xi}\left[\lim _{T \rightarrow \infty} \frac{1}{T} \sum_{t=1}^{T} I_{t}^{a}\right]=\lim _{T \rightarrow \infty} \frac{1}{T} \sum_{t=1}^{T} \mathbb{E}_{s}^{\xi}\left[I_{t}^{a}\right]$ and thus freq $(\xi, s, a)=$ $\frac{\bar{x}_{a}}{\bar{x}_{D}}=$ freq $(\xi, D, a)$. This finishes the proof of Lemma 4.3.

Assume that the system $L$ is solved by assigning nonnegative values $\bar{x}_{a}$ to $x_{a}$ and $\bar{y}_{\chi}$ to $y_{\chi}$ where $\chi \in A \cup S$. W.l.o.g. assume that $\bar{y}_{s}=0$ for all states $s$ not contained in any MEC. Let $\xi$ be the strategy of Lemma 4.3, Using Equations (4.1), (4.2), and (4.3), we will define a 2-memory stochastic update strategy $\sigma$ as follows. The strategy $\sigma$ has two memory elements, $m_{1}$ and $m_{2}$. A run of $G^{\sigma}$ starts in $s_{0}$ with a given distribution on memory elements (see below). Then $\sigma$ plays according to a suitable memoryless strategy (constructed below) until the memory changes to $m_{2}$, and then it starts behaving as $\xi$ forever. Given a BSCC $D$ of $G^{\xi}$, we denote by $\mathbb{P}_{s_{0}}^{\sigma}$ [switch to $\xi$ in $D$ ] the probability that $\sigma$ switches from $m_{1}$ to $m_{2}$ while in $D$. We construct $\sigma$ so that

$$
\mathbb{P}_{s_{0}}^{\sigma}[\text { switch to } \xi \text { in } D]=\sum_{a \in D \cap A} \bar{x}_{a}
$$

Then for all $a \in D \cap A$ we have freq $\left(\sigma, s_{0}, a\right)=\mathbb{P}_{s_{0}}^{\sigma}[\operatorname{switch}$ to $\xi$ in $D] \cdot \operatorname{freq}(\xi, D, a)=\bar{x}_{a}$. Finally, we obtain the following:

$$
\mathbb{E}_{s_{0}}^{\sigma}\left[\operatorname{lr}_{\mathrm{inf}}\left(\vec{r}_{i}\right)\right]=\sum_{a \in A} \vec{r}_{i}(a) \cdot \bar{x}_{a}
$$


The equation can be derived as follows:

$$
\begin{array}{rlr}
\mathbb{E}_{s_{0}}^{\sigma}\left[\operatorname{lr}_{\mathrm{inf}}\left(r_{i}\right)\right] & =\mathbb{E}_{s_{0}}^{\sigma}\left[\liminf _{T \rightarrow \infty} \frac{1}{T} \sum_{t=1}^{T} r_{i}\left(A_{t}\right)\right] & \text { (definition) } \\
& =\mathbb{E}_{s_{0}}^{\sigma}\left[\lim _{T \rightarrow \infty} \frac{1}{T} \sum_{t=1}^{T} r_{i}\left(A_{t}\right)\right] & \text { (see below) } \\
& =\lim _{T \rightarrow \infty} \frac{1}{T} \sum_{t=1}^{T} \mathbb{E}_{s_{0}}^{\sigma}\left[r_{i}\left(A_{t}\right)\right] & \text { (see below) } \\
& =\lim _{T \rightarrow \infty} \frac{1}{T} \sum_{t=1}^{T} \sum_{a \in A} r_{i}(a) \cdot \mathbb{P}_{s_{0}}^{\sigma}\left[A_{t}=a\right] & \text { (definition of expectation) } \\
& =\sum_{a \in A} r_{i}(a) \cdot \lim _{T \rightarrow \infty} \frac{1}{T} \sum_{t=1}^{T} \mathbb{P}_{s_{0}}^{\sigma}\left[A_{t}=a\right] \\
& =\sum_{a \in A} r_{i}(a) \cdot \operatorname{freq}\left(\sigma, s_{0}, a\right) & \text { (linearity of the limit) } \\
& =\sum_{a \in A} r_{i}(a) \cdot \bar{x}_{a}
\end{array}
$$

The second equality follows from the fact that the limit is almost surely defined, following from the Ergodic theorem applied to the BSCCs of the finite Markov chain $G^{\sigma}$. The third equality holds by Lebesgue Dominated convergence theorem, because $\left|r_{i}\left(A_{t}\right)\right| \leq$ $\max _{a \in A}\left|r_{i}(a)\right|$.

Note that the right-hand side of Equation (4.7) is greater than or equal to $\vec{v}_{i}$ by Inequality (4.5) of $L$.

So, it remains to construct the strategy $\sigma$ with the desired "switching" property expressed by Equations (4.6). Roughly speaking, we proceed in two steps.

1. We construct a finite-memory stochastic update strategy $\bar{\sigma}$ satisfying Equations (4.6). The strategy $\bar{\sigma}$ is constructed so that it initially behaves as a certain finite-memory stochastic update strategy, but eventually this mode is "switched" to the strategy $\xi$ which is followed forever.

2. The only problem with $\bar{\sigma}$ is that it may use more than two memory elements in general. This is solved by applying the results of [10] and reducing the "initial part" of $\bar{\sigma}$ (i.e., the part before the switch) into a memoryless strategy. Thus, we transform $\bar{\sigma}$ into an "equivalent" strategy $\sigma$ which is 2-memory stochastic update.

Now we elaborate the two steps.

Step 1. For every MEC $C$ of $G$, we denote by $y_{C}$ the number $\sum_{s \in C} \bar{y}_{s}=\sum_{a \in A \cap C} \bar{x}_{a}$. By combining the solution of $L$ with the results of Sections 3 and 5 of [10] one can construct a finite-memory stochastic-update strategy $\zeta$ which stays eventually in each MEC $C$ with probability $y_{C}$. Formally, the construction is captured in the following lemma.

Lemma 4.4. Consider numbers $\bar{y}_{\chi}$ for all $\chi \in S \cup A$ such that the assignment $y_{\chi}:=\bar{y}_{\chi}$ is a part of some nonnegative solution to $L$. Then there is a finite-memory stochastic update strategy $\zeta$ which, starting from $s_{0}$, stays eventually in each $M E C C$ with probability $y_{C}:=\sum_{s \in C} \bar{y}_{s}$. 
Proof. In order to be able to use results of [10, Section 3] we modify the MDP $G$ and obtain a new MDP $G^{\prime}$ as follows: For each state $s$ we add a new absorbing state, $d_{s}$. The only available action for $d_{s}$ leads to a loop transition back to $d_{s}$ with probability 1 . We also add a new action, $a_{s}^{d}$, to every $s \in S$. The distribution associated with $a_{s}^{d}$ assigns probability 1 to $d_{s}$.

Let us call $K$ the set of constraints of the LP on Figure 3 in [10]. From the values $\bar{y}_{\chi}$ we now construct a solution to $K$ : for every state $s \in S$ and every action $a \in \operatorname{Act}(s)$ we set $y_{(s, a)}:=\bar{y}_{a}$, and $y_{\left(s, a_{s}^{d}\right)}:=\bar{y}_{s}$. The values of the rest of variables in $K$ are determined by the second set of equations in $K$. The nonnegative constraints in $K$ are satisfied since $\bar{y}_{\chi}$ are nonnegative. Finally, the equations (4.1) from $L$ imply that the first set of equations in $K$ are satisfied, because $\bar{y}_{\chi}$ are part of a solution to $L$.

By Theorem 3.2 of [10] we thus have a memoryless strategy $\varrho$ for $G^{\prime}$ which satisfies $\mathbb{P}_{\varrho}^{s_{0}}\left[\right.$ Reach $\left.\left(d_{s}\right)\right] \geq y_{s}$ for all $s \in S$. The strategy $\zeta$ then mimics the behavior of $\varrho$ until the moment when $\varrho$ chooses an action to enter some of the new absorbing states. From that point on, $\zeta$ may choose some arbitrary fixed behavior to stay in the current MEC (note that if the current state $s$ is not included in any MEC, then $\bar{y}_{s}=0$ and so the strategy $\varrho$ would not choose to enter the new absorbing state). As a consequence: $\mathbb{P}_{\zeta}^{s_{0}}[$ stay eventually in $C] \geq y_{C}$, and in fact, we get equality here, because of the equations (4.2) from $L$. Note that $\zeta$ only needs a finite constant amount of memory.

The strategy $\bar{\sigma}$ works as follows. For a run initiated in $s_{0}$, the strategy $\bar{\sigma}$ plays according to $\zeta$ until a BSCC of $G^{\zeta}$ is reached. This means that every possible continuation of the path stays in the current MEC $C$ of $G$. Assume that $C$ has states $s_{1}, \ldots, s_{k}$. We denote by $\bar{x}_{s}$ the sum $\sum_{a \in A c t(s)} \bar{x}_{a}$. At this point, the strategy $\bar{\sigma}$ changes its behavior as follows: First, the strategy $\bar{\sigma}$ strives to reach $s_{1}$ with probability one. Upon reaching $s_{1}$, it chooses (randomly, with probability $\frac{\bar{x}_{s_{1}}}{y_{C}}$ ) either to behave as $\xi$ forever, or to follow on to $s_{2}$. If the strategy $\bar{\sigma}$ chooses to go on to $s_{2}$, it strives to reach $s_{2}$ with probability one. Upon reaching $s_{2}$, the strategy $\bar{\sigma}$ chooses (randomly, with probability $\frac{\bar{x}_{s_{2}}}{y_{C}-\bar{x}_{s_{1}}}$ ) either to behave as $\xi$ forever, or to follow on to $s_{3}$, and so, till $s_{k}$. That is, the probability of switching to $\xi$ in $s_{i}$ is $\frac{\bar{x}_{s_{i}}}{y_{C}-\sum_{j=1}^{i-1} \bar{x}_{s_{j}}}$.

Since $\zeta$ stays in a MEC $C$ with probability $y_{C}$, the probability that the strategy $\bar{\sigma}$ switches to $\xi$ in $s_{i}$ is equal to $\bar{x}_{s_{i}}$. However, then for every BSCC $D$ of $G^{\xi}$ satisfying $D \cap C \neq \emptyset$ (and thus $D \subseteq C$ ) we have that the strategy $\bar{\sigma}$ switches to $\xi$ in a state of $D$ with probability $\sum_{s \in D \cap S} \bar{x}_{s}=\sum_{a \in D \cap A} \bar{x}_{a}$. Hence, $\bar{\sigma}$ satisfies Equations (4.6).

Step 2. Now we show how to reduce the first phase of $\bar{\sigma}$ (before the switch to $\xi$ ) into a memoryless strategy, using the results of [10, Section 3]. Unfortunately, these results are not applicable directly. We need to modify the MDP $G$ into a new MDP $G^{\prime}$, same as we did above: For each state $s$ we add a new absorbing state, $d_{s}$. The only available action for $d_{s}$ leads to a loop transition back to $d_{s}$ with probability 1 . We also add a new action, $a_{s}^{d}$, to every $s \in S$. The distribution associated with $a_{s}^{d}$ assigns probability 1 to $d_{s}$.

Let us consider a finite-memory stochastic-update strategy, $\sigma^{\prime}$, for $G^{\prime}$ defined as follows. The strategy $\sigma^{\prime}$ behaves as $\bar{\sigma}$ before the switch to $\xi$. Once $\bar{\sigma}$ switches to $\xi$, say in a state $s$ of $G$ with probability $p_{s}$, the strategy $\sigma^{\prime}$ chooses the action $a_{s}^{d}$ with probability $p_{s}$. It follows that the probability of $\bar{\sigma}$ switching in $s$ is equal to the probability of reaching $d_{s}$ in $G^{\prime}$ under $\sigma^{\prime}$. By [10, Theorem 3.2], there is a memoryless strategy, $\sigma^{\prime \prime}$, for $G^{\prime}$ that reaches $d_{s}$ with probability $p_{s}$. We define $\sigma$ in $G$ to behave as $\sigma^{\prime \prime}$ with the exception that, in every 
state $s$, instead of choosing an action $a_{s}^{d}$ with probability $p_{s}$ it switches to behave as $\xi$ with probability $p_{s}$ (which also means that the initial distribution on memory elements assigns $p_{s_{0}}$ to $m_{2}$ ). Then, clearly, $\sigma$ satisfies Equations (4.6) because

$$
\mathbb{P}_{s_{0}}^{\sigma}[\text { switch in } D]=\sum_{s \in D} \mathbb{P}_{s_{0}}^{\sigma^{\prime \prime}}\left[\text { fire } a_{s}^{d}\right]=\sum_{s \in D} \mathbb{P}_{s_{0}}^{\sigma^{\prime}}\left[\text { fire } a_{s}^{d}\right]=\mathbb{P}_{s_{0}}^{\bar{\sigma}}[\text { switch in } D]=\sum_{a \in D \cap A} \bar{x}_{a} \text {. }
$$

This concludes the proof of Proposition 4.2 .

Proposition 4.5. If $\vec{v} \in \operatorname{AcEx}\left(\operatorname{lr}_{\mathrm{inf}}(\vec{r})\right)$, then $L$ has a nonnegative solution.

Proof. Let $\varrho \in \Sigma$ be a strategy such that $\mathbb{E}_{s_{0}}^{\varrho}\left[\operatorname{lr}_{\text {inf }}(\vec{r})\right] \geq \vec{v}$. In general, the frequencies freq $\left(\varrho, s_{0}, a\right)$ of the actions may not be well defined, because the defining limits may not exist. A crucial trick to overcome this difficulty is to pick suitable "related" values, $f(a)$, lying between $\liminf _{T \rightarrow \infty} \frac{1}{T} \sum_{t=1}^{T} \mathbb{P}_{s_{0}}^{\varrho}\left[A_{t}=a\right]$ and $\limsup _{T \rightarrow \infty} \frac{1}{T} \sum_{t=1}^{T} \mathbb{P}_{S_{0}}^{\varrho}\left[A_{t}=a\right]$, which can be safely substituted for $x_{a}$ in $L$. Since every infinite sequence contains an infinite convergent subsequence, there is an increasing sequence of indices, $T_{0}, T_{1}, \ldots$, such that the following limit exists for each action $a \in A$

$$
f(a):=\lim _{\ell \rightarrow \infty} \frac{1}{T_{\ell}} \sum_{t=1}^{T_{\ell}} \mathbb{P}_{s_{0}}^{\varrho}\left[A_{t}=a\right] .
$$

Setting $x_{a}:=f(a)$ for all $a \in A$ satisfies Inequalities (4.5) and Equations (4.4) of $L$. Indeed, the former follows from $\mathbb{E}_{s_{0}}^{\varrho}\left[\operatorname{lr}_{\text {inf }}(\vec{r})\right] \geq \vec{v}$ and the following inequality, which holds for all $1 \leq i \leq k$ :

$$
\sum_{a \in A} \vec{r}_{i}(a) \cdot f(a) \geq \mathbb{E}_{s_{0}}^{\varrho}\left[\operatorname{lr}_{\mathrm{inf}}\left(\vec{r}_{i}\right)\right]
$$

The inequality follows from the following derivation:

$$
\begin{array}{rlr}
\sum_{a \in A} r_{i}(a) \cdot f(a) & =\sum_{a \in A} r_{i}(a) \cdot \lim _{\ell \rightarrow \infty} \frac{1}{T_{\ell}} \sum_{t=1}^{T_{\ell}} \mathbb{P}_{s_{0}}^{\varrho}\left[A_{t}=a\right] & \text { (definition of } f(a)) \\
& =\lim _{\ell \rightarrow \infty} \frac{1}{T_{\ell}} \sum_{t=1}^{T_{\ell}} \sum_{a \in A} r_{i}(a) \cdot \mathbb{P}_{s_{0}}^{\varrho}\left[A_{t}=a\right] & \text { (linearity of the limit) } \\
& \geq \liminf _{T \rightarrow \infty} \frac{1}{T} \sum_{t=1}^{T} \sum_{a \in A} r_{i}(a) \cdot \mathbb{P}_{s_{0}}^{\varrho}\left[A_{t}=a\right] & \text { (definition of lim inf) } \\
& \geq \liminf _{T \rightarrow \infty} \frac{1}{T} \sum_{t=1}^{T} \mathbb{E}_{s_{0}}^{\varrho}\left[r_{i}\left(A_{t}\right)\right] \\
& \geq \mathbb{E}_{s_{0}}^{\varrho}\left[\operatorname{lr}_{\inf }\left(r_{i}\right)\right] . & \text { (linearity of the expectation) }
\end{array}
$$

The last inequality is a consequence of Fatou's lemma (see, e.g. [19, Chapter 4, Section 3]) although the function $r_{i}\left(A_{t}\right)$ may not be nonnegative, we can replace it with the nonnegative function $r_{i}\left(A_{t}\right)-\min _{a \in A} r_{i}(a)$ and add the subtracted constant afterwards.

To prove that Equations (4.4) are satisfied, it suffices to show that for all $s \in S$ we have

$$
\sum_{a \in A} f(a) \cdot \delta(a)(s)=\sum_{a \in A c t(s)} f(a)
$$


This holds, because

$$
\begin{aligned}
& \sum_{a \in A} f(a) \cdot \delta(a)(s)=\sum_{a \in A} \lim _{\ell \rightarrow \infty} \frac{1}{T_{\ell}} \sum_{t=1}^{T_{\ell}} \mathbb{P}_{s_{0}}^{\varrho}\left[A_{t}=a\right] \cdot \delta(a)(s) \\
& =\lim _{\ell \rightarrow \infty} \frac{1}{T_{\ell}} \sum_{t=1}^{T_{\ell}} \sum_{a \in A} \mathbb{P}_{s_{0}}^{\varrho}\left[A_{t}=a\right] \cdot \delta(a)(s) \quad \text { (linearity of the limit) } \\
& =\lim _{\ell \rightarrow \infty} \frac{1}{T_{\ell}} \sum_{t=1}^{T_{\ell}} \mathbb{P}_{s_{0}}^{\varrho}\left[S_{t+1}=s\right] \\
& =\lim _{\ell \rightarrow \infty} \frac{1}{T_{\ell}} \sum_{t=1}^{T_{\ell}} \mathbb{P}_{s_{0}}^{\varrho}\left[S_{t}=s\right] \\
& =\lim _{\ell \rightarrow \infty} \frac{1}{T_{\ell}} \sum_{t=1}^{T_{\ell}} \sum_{a \in A c t(s)} \mathbb{P}_{s_{0}}^{\varrho}\left[A_{t}=a\right] \quad(s \text { must be followed by } a \in \operatorname{Act}(s)) \\
& =\sum_{a \in A c t(s)} \lim _{\ell \rightarrow \infty} \frac{1}{T_{\ell}} \sum_{t=1}^{T_{\ell}} \mathbb{P}_{s_{0}}^{\varrho}\left[A_{t}=a\right] \quad \text { (linearity of the limit) } \\
& =\sum_{a \in \operatorname{Act}(s)} f(a) .
\end{aligned}
$$

The fourth equality follows from the following:

$$
\begin{aligned}
\lim _{\ell \rightarrow \infty} \frac{1}{T_{\ell}} \sum_{t=1}^{T_{\ell}} \mathbb{P}_{s_{0}}^{\varrho}\left[S_{t+1}=s\right]-\lim _{\ell \rightarrow \infty} \frac{1}{T_{\ell}} \sum_{t=1}^{T_{\ell}} \mathbb{P}_{s_{0}}^{\varrho}\left[S_{t}=s\right] & =\lim _{\ell \rightarrow \infty} \frac{1}{T_{\ell}} \sum_{t=1}^{T_{\ell}}\left(\mathbb{P}_{s_{0}}^{\varrho}\left[S_{t+1}=s\right]-\mathbb{P}_{s_{0}}^{\varrho}\left[S_{t}=s\right]\right) \\
& =\lim _{\ell \rightarrow \infty} \frac{1}{T_{\ell}}\left(\mathbb{P}_{s_{0}}^{\varrho}\left[S_{T_{\ell}+1}=s\right]-\mathbb{P}_{s_{0}}^{\varrho}\left[S_{1}=s\right]\right) \\
& =0 .
\end{aligned}
$$

Now we have to set the values for $y_{\chi}, \chi \in A \cup S$, and prove that they satisfy the rest of $L$ when the values $f(a)$ are assigned to $x_{a}$. Note that almost every run of $G^{\varrho}$ eventually stays in some MEC of $G$ (cf., e.g., [9, Proposition 3.1]). For every MEC $C$ of $G$, let $y_{C}$ be the probability of all runs in $G^{e}$ that eventually stay in $C$. Note that

$$
\begin{aligned}
\sum_{a \in A \cap C} f(a) & =\sum_{a \in A \cap C} \lim _{\ell \rightarrow \infty} \frac{1}{T_{\ell}} \sum_{t=1}^{T_{\ell}} \mathbb{P}_{s_{0}}^{\varrho}\left[A_{t}=a\right] \\
& =\lim _{\ell \rightarrow \infty} \frac{1}{T_{\ell}} \sum_{t=1}^{T_{\ell}} \sum_{a \in A \cap C} \mathbb{P}_{s_{0}}^{\varrho}\left[A_{t}=a\right] \\
& =\lim _{\ell \rightarrow \infty} \frac{1}{T_{\ell}} \sum_{t=1}^{T_{\ell}} \mathbb{P}_{s_{0}}^{\varrho}\left[A_{t} \in C\right]=y_{C} .
\end{aligned}
$$

Here the last equality follows from the fact that $\lim _{\ell \rightarrow \infty} \mathbb{P}_{s_{0}}^{\varrho}\left[A_{T_{\ell}} \in C\right]$ is equal to the probability of all runs in $G^{\varrho}$ that eventually stay in $C$ (recall that almost every run stays eventually in a MEC of $G$ ) and the fact that the Cesàro sum of a convergent sequence is equal to the limit of the sequence. 
To obtain $y_{a}$ and $y_{s}$, we need to simplify the behavior of $\varrho$ before reaching a MEC for which we use the results of [10]. As in the proof of Proposition 4.2, we first need to modify the MDP $G$ into another MDP $G^{\prime}$ as follows: For each state $s$ we add a new absorbing state, $d_{s}$. The only available action for $d_{s}$ leads to a loop transition back to $d_{s}$ with probability 1 . We also add a new action, $a_{s}^{d}$, to every $s \in S$. The distribution associated with $a_{s}^{d}$ assigns probability 1 to $d_{s}$. Using the results of [10], we prove the following lemma.

Lemma 4.6. The existence of a strategy @ satisfying $\mathbb{E}_{s_{0}}^{\varrho}\left[\operatorname{lr}_{\text {inf }}(\vec{r})\right] \geq \vec{v}$ implies the existence of a (possibly randomized) memoryless strategy $\zeta$ for $G^{\prime}$ such that

$$
\sum_{s \in C} \mathbb{P}_{s_{0}}^{\zeta}\left[\operatorname{Reach}\left(d_{s}\right)\right]=y_{C} .
$$

Proof. We give a proof by contradiction. Note that the proof structure is similar to the proof of direction $3 \Rightarrow 1$ of Theorem 3.2 in [10]. Let $C_{1}, \ldots C_{n}$ be all MECs of $G$, and let $X \subseteq \mathbb{R}^{n}$ be the set of all vectors $\left(x_{1}, \ldots, x_{n}\right)$ for which there is a strategy $\bar{\sigma}$ in $G^{\prime}$ such that $\mathbb{P}_{s_{0}}^{\bar{\sigma}}\left[\bigcup_{s \in C_{i}} \operatorname{Reach}\left(d_{s}\right)\right] \geq x_{i}$ for all $1 \leq i \leq n$. For a contradiction, suppose $\left(y_{C_{1}}, \ldots, y_{C_{n}}\right) \notin X$. By [10, Theorem 3.2] the set $X$ can be described as a set of solutions of a linear program, and hence it is convex. By the separating hyperplane theorem 2] there are weights $w_{1}, \ldots, w_{n}$ such that $\sum_{i=1}^{n} y_{C_{i}} \cdot w_{i}>\sum_{i=1}^{n} x_{i} \cdot w_{i}$ for every $\left(x_{1}, \ldots, x_{n}\right) \in X$.

We define a reward function $r$ by $r(a)=w_{i}$ for an action $a$ from $C_{i}$, where $1 \leq i \leq n$, and $r(a)=0$ for actions not in any MEC. Observe that the mean payoff of any run that eventually stays in a MEC $C_{i}$ is $w_{i}$, and so the expected mean payoff w.r.t. $r$ under $\varrho$ is $\sum_{i=1}^{n} y_{C_{i}} \cdot w_{i}$. Because memoryless deterministic strategies suffice for maximising the (single-objective) expected mean payoff, there is also a memoryless deterministic strategy $\hat{\sigma}$ for $G$ that yields expected mean payoff w.r.t. $r$ equal to $z \geq \sum_{i=1}^{n} y_{C_{i}} \cdot w_{i}$. We now define a strategy $\bar{\sigma}$ for $G^{\prime}$ to mimic $\hat{\sigma}$ until a BSCC is reached, and when a BSCC is reached, say along a path $w$, the strategy $\bar{\sigma}$ takes the action $a_{\text {last }(w)}^{d}$. Let $x_{i}=\mathbb{P}_{s_{0}}^{\bar{\sigma}}\left[\bigcup_{s \in C_{i}} \operatorname{Reach}\left(d_{s}\right)\right]$. Due to the construction of $\bar{\sigma}$ we have $x_{i}$ is equal to the probability of runs that eventually stay in $C_{i}$ under $\hat{\sigma}$ : this follows because once a BSCC is reached on a path $w$, every run $\omega$ extending $w$ has an infinite suffix containing only states from the MEC containing the state $\operatorname{last}(w)$. Hence $\sum_{i=1}^{n} x_{i} \cdot w_{i}=z$. However, by the choice of the weights $w_{i}$ we get that $\left(x_{1}, \ldots, x_{n}\right) \notin X$, and hence a contradiction, because $\bar{\sigma}$ witnesses that $\left(x_{1}, \ldots, x_{n}\right) \in X$.

Hence, we have obtained that there is some (possibly memory-dependent) strategy $\zeta$, and using [10, Theorem 3.2] we get that there also is a memoryless strategy $\zeta$ with the required properties. This completes the proof of Lemma 4.6.

We now proceed with the proof of Proposition 4.5. Let $U_{a}$ be a function over the runs in $G^{\prime}$ returning the (possibly infinite) number of times the action $a$ is used. We are now ready to define the assignment for the variables $y_{\chi}$ of $L$.

$$
\begin{array}{ll}
y_{a}:=\mathbb{E}_{s_{0}}^{\zeta}\left[U_{a}\right] & \text { for all } a \in A \\
y_{s}:=\mathbb{E}_{s_{0}}^{\zeta}\left[U_{a_{s}^{d}}\right]=\mathbb{P}_{s_{0}}^{\zeta}\left[\operatorname{Reach}\left(d_{s}\right)\right] & \text { for all } s \in S .
\end{array}
$$

Note that [10, Lemma 3.3] ensures that all $y_{a}$ and $y_{s}$ are indeed well-defined finite values, and satisfy Equations (4.1) of $L$. Equations (4.3) of $L$ are satisfied due to Equations (4.11) and (4.10). Equations (4.11) together with $\sum_{a \in A \cap C} f(a)=1$ imply Equations (4.2) of $L$. This completes the proof of Proposition 4.5. 
The item A.1 in Section 3.1 follows directly from Theorem 4.1, Let us analyze A.2. Suppose $\vec{v}$ is a point of the Pareto curve. Consider the system $L^{\prime}$ of linear inequalities obtained from $L$ by replacing constants $\vec{v}_{i}$ in Inequalities (4.5) with new variables $z_{i}$. Let $Q \subseteq \mathbb{R}^{n}$ be the projection of the set of solutions of $L^{\prime}$ to $z_{1}, \ldots, z_{n}$. From Theorem 4.1 and the definition of Pareto curve, the (Euclidean) distance of $\vec{v}$ to $Q$ is 0 . Because the set of solutions of $L^{\prime}$ is a closed set, $Q$ is also closed and thus $\vec{v} \in Q$. This gives us a solution to $L$ with variables $z_{i}$ having values $\vec{v}_{i}$, and we can use Theorem 4.1 to get a strategy witnessing that $\vec{v} \in \operatorname{AcEx}\left(\operatorname{lr}_{\text {inf }}(\vec{r})\right)$.

Now consider the items A.3 and A.4. The system $L$ is linear, and hence the problem whether $\vec{v} \in \operatorname{AcEx}\left(\operatorname{lr}_{\inf }(\vec{r})\right)$ is decidable in polynomial time by employing polynomial-time algorithms for linear programming. A 2-memory stochastic-update strategy $\sigma$ satisfying $\mathbb{E}_{s}^{\sigma}\left[\operatorname{lr}_{\text {inf }}(\vec{r})\right] \geq \vec{v}$ can be computed as follows (note that the proof of Proposition 4.2 is not fully constructive, so we cannot apply this proposition immediately). First, we find a solution of the system $L$, and we denote by $\bar{x}_{a}$ the value assigned to $x_{a}$. Let $\left(T_{1}, B_{1}\right), \ldots,\left(T_{n}, B_{n}\right)$ be the end components such that $a \in \bigcup_{i=1}^{n} B_{i}$ iff $\bar{x}_{a}>0$, and $T_{1}, \ldots, T_{n}$ are pairwise disjoint. We construct another system of linear inequalities consisting of Equations (1) of $L$ and the equations $\sum_{s \in T_{i}} y_{s}=\sum_{s \in T_{i}} \sum_{a \in A c t(s)} \bar{x}_{a}$ for all $1 \leq i \leq n$. Due to [10], there is a solution to this system iff in the MDP $G^{\prime}$ from the proof of Proposition 4.2 there is a strategy that for every $i$ reaches $d_{s}$ for $s \in T_{i}$ with probability $\sum_{s \in T_{i}} \sum_{a \in A c t(s)} \bar{x}_{a}$. Such a strategy indeed exists (consider, e.g., the strategy $\sigma^{\prime}$ from the proof of Proposition 4.2). Thus, there is a solution to the above system and we can denote by $\hat{y}_{s}$ and $\hat{y}_{a}$ the values assigned to $y_{s}$ and $y_{a}$. We define $\sigma$ by

$$
\begin{aligned}
& \sigma_{n}\left(s, m_{1}\right)(a)=\bar{y}_{a} / \sum_{a^{\prime} \in \operatorname{Act}(s)} \bar{y}_{a^{\prime}} \\
& \sigma_{n}\left(s, m_{2}\right)(a)=\bar{x}_{a} / \sum_{a^{\prime} \in \operatorname{Act}(s)} \bar{x}_{a^{\prime}}
\end{aligned}
$$

and further $\sigma_{u}\left(a, s, m_{1}\right)\left(m_{2}\right)=y_{s}, \sigma_{u}\left(a, s, m_{2}\right)\left(m_{2}\right)=1$, and the initial memory distribution assigns $\left(1-y_{s_{0}}\right)$ and $y_{s_{0}}$ to $m_{1}$ and $m_{2}$, respectively. Due to [10] we have

$$
\mathbb{P}_{s_{0}}^{\sigma}\left[\text { change memory to } m_{2} \text { in } s\right]=\hat{y}_{s},
$$

and the rest follows similarly as in the proof of Proposition 4.2 .

The item A.5 can be proved as follows: To test that $\vec{v} \in \operatorname{AcEx}\left(\operatorname{lr}_{\text {inf }}(\vec{r})\right)$ lies in the Pareto curve we turn the system $L$ into a linear program $L P$ by adding the objective to maximize $\sum_{1 \leq i \leq k} \sum_{a \in A} x_{a} \cdot \vec{r}_{i}(a)$. Then we check that there is no better solution than $\sum_{1 \leq i \leq k} \vec{v}_{i}$.

Finally, the item A.6 is obtained by considering the system $L^{\prime}$ above and computing all exponentially many vertices of the polytope of all solutions. Then we compute projections of these vertices onto the dimensions $z_{1}, \ldots, z_{n}$ and retrieve all the maximal vertices. Moreover, if for every $\vec{v} \in\left\{\ell \cdot \varepsilon \mid \ell \in \mathbb{Z} \wedge-M_{r} \leq \ell \cdot \varepsilon \leq M_{r}\right\}^{k}$ where $M_{r}=\max _{a \in A} \max _{1 \leq i \leq k}\left|\vec{r}_{i}(a)\right|$ we decide whether $\vec{v} \in \operatorname{AcEx}\left(\operatorname{lr}_{\text {inf }}(\vec{r})\right)$, we can easily construct an $\varepsilon$-approximate Pareto curve.

4.1. Deterministic-update Strategies for Expectation Objectives. We now show that for expectation objectives, finite-memory deterministic update strategies suffice. This is captured in the following proposition.

Proposition 4.7. Every nonnegative solution of the system $L$ induces a finite-memory deterministic-update strategy $\sigma$ satisfying $\mathbb{E}_{s_{0}}^{\sigma}\left[\operatorname{lr}_{\text {inf }}(\vec{r})\right] \geq \vec{v}$.

Proof. The proof proceeds almost identically to the proof of Proposition 4.2. Let us recall the important steps from that proof first. There we worked with the numbers $\bar{x}_{a}, a \in A$, 
which, assigned to the variables $x_{a}$, formed a part of the solution to $L$. We also worked with two important strategies. The first one, a finite-memory deterministic-update strategy $\zeta$, made sure that, starting in $s_{0}$, a run stays in a MEC $C$ forever with probability $y_{C}=$ $\sum_{a \in A \cap C} \bar{x}_{a}$. The second one, a memoryless strategy $\sigma^{\prime}$, had the property that when the starting distribution was $\alpha(s):=\bar{x}_{s}=\sum_{a \in A c t(s)} \bar{x}_{a}$ then $\mathbb{E}_{\alpha}^{\sigma^{\prime}}\left[\operatorname{lr}_{\text {inf }}(\vec{r})\right] \geq \vec{v}$. 11 To produce the promised finite-memory deterministic-update strategy $\sigma$ we now have to combine the strategies $\zeta$ and $\sigma^{\prime}$ using only deterministic memory updates.

We now define the strategy $\sigma$. It works in three phases. First, it reaches every MEC $C$ and stays in it with the probability $y_{C}$. Second, it prepares the distribution $\alpha$, and finally third, it switches to $\sigma^{\prime}$. It is clear how the strategy is defined in the third phase. As for the first phase, this is also identical to what we did in the proof of Proposition 4.2 for $\bar{\sigma}$ : The strategy $\sigma$ follows the strategy $\zeta$ from beginning until in the associated finite state Markov chain $G^{\zeta}$ a bottom strongly connected component (BSCC) is reached. At that point the run has already entered its final MEC $C$ to stay in it forever, which happens with probability $y_{C}$.

The last thing to solve is thus the second phase. Two cases may occur. Either there is a state $s \in C$ such that $|A c t(s) \cap C|>1$, i.e., there are at least two actions the strategy can take from $s$ without leaving $C$. Let us denote these actions $a$ and $b$. Consider an enumeration $C=\left\{s_{1}, \ldots, s_{k}\right\}$ of vertices of $C$. Now we define the second phase of $\sigma$ when in $C$. We start with defining the memory used in the second phase. We symbolically represent the possible contents of the memory as $\left\{\right.$ Wait $_{1}, \ldots$, Wait $_{k}$, Switch $_{1}, \ldots$, Switch $\left._{k}\right\}$. The second phase then starts with the memory set to Wait ${ }_{1}$. Generally, if the memory is set to Wait ${ }_{i}$ then $\sigma$ aims at reaching $s$ with probability 1 . This is possible (since $s$ is in the same MEC) and it is a well known fact that it can be done without using memory. On visiting $s$, the strategy chooses the action $a$ with probability $\bar{x}_{s_{i}} /\left(y_{C}-\sum_{j=1}^{i-1} \bar{x}_{s_{j}}\right)$ and the action $b$ with the remaining probability. In the next step the deterministic update function sets the memory either to $\mathrm{Switch}_{i}$ or $\mathrm{Wait}_{i+1}$, depending on whether the last action seen is $a$ or $b$, respectively. (Observe that if $i=k$ then the probability of taking $b$ is 0 .) The memory set to Switch $_{i}$ means that the strategy aims at reaching $s_{i}$ almost surely, and upon doing so, the strategy switches to the third phase, following $\sigma^{\prime}$. It is easy to observe that on the condition of staying in $C$ the probability of switching to the third phase in some $s_{i} \in C$ is $\bar{x}_{s_{i}} / y_{C}$, thus the unconditioned probability of doing so is $\bar{x}_{s_{i}}$, as desired.

The remaining case to solve is when $|\operatorname{Act}(s) \cap C|=1$ for all $s \in C$. But then switching to the third phase is solved trivially with the right probabilities, because staying in $C$ inevitably already means mimicking $\sigma^{\prime}$.

\section{Solution for Satisfaction Objectives}

In this section we prove the items B.1-B.6 of Section 3.2. Let us fix an MDP $G$, a vector of rewards, $\vec{r}=\left(r_{1}, \ldots, r_{k}\right)$, and an initial state $s_{0}$. We start by assuming that the MDP $G$ is strongly connected (i.e., $(S, A)$ is an end component).

Proposition 5.1. Assume that $G$ is strongly connected and that there is a strategy $\pi$ such that $\mathbb{P}_{s_{0}}^{\pi}\left[\operatorname{lr}_{\inf }(\vec{r}) \geq \vec{v}\right]>0$. Then the following is true.

\footnotetext{
${ }^{1}$ Here we extend the notation in a straightforward way from a single initial state to a general initial distribution, $\alpha$.
} 
1. There is a strategy $\xi$ satisfying $\mathbb{P}_{s}^{\xi}\left[\operatorname{lr}_{\text {inf }}(\vec{r}) \geq \vec{v}\right]=1$ for all $s \in S$.

2. For each $\varepsilon>0$ there is a memoryless randomized strategy $\xi_{\varepsilon}$ that for all $s \in S$ satisfies $\mathbb{P}_{s}^{\xi_{\varepsilon}}\left[\operatorname{lr}_{\text {inf }}(\vec{r}) \geq \vec{v}-\vec{\varepsilon}\right]=1$.

Moreover, the problem whether there is some $\pi$ such that $\mathbb{P}_{s_{0}}^{\pi}\left[\operatorname{lr}_{\mathrm{inf}}(\vec{r}) \geq \vec{v}\right]>0$ is decidable in polynomial time. Strategies $\xi_{\varepsilon}$ are computable in time polynomial in the size of $G$, the size of the binary representation of $\vec{r}$, and $\frac{1}{\varepsilon}$.

Proof. By [6, 13] we get that $\mathbb{P}_{s_{0}}^{\pi}\left[\operatorname{lr}_{\text {inf }}(\vec{r}) \geq \vec{v}\right]>0$ implies that there is a strategy $\xi$ such that $\mathbb{P}_{s_{0}}^{\xi}\left[\operatorname{lr}_{\text {inf }}(\vec{r}) \geq \vec{v}\right]=1$ : Since $\operatorname{lr}_{\text {inf }}(\vec{r}) \geq \vec{v}$ is a tail or prefix-independent function, it follows from the results of [6] that if $\mathbb{P}_{s_{0}}^{\pi}\left[\operatorname{lr}_{\text {inf }}(\vec{r}) \geq \vec{v}\right]>0$, then there exists a state $s$ in the MDP with value 1 , i.e., there exists $s$ such that $\sup _{\pi} \mathbb{P}_{s}^{\pi}\left[\operatorname{lr}_{\text {inf }}(\vec{r}) \geq \vec{v}\right]=1$. It follows from the results of [13] that in MDPs with tail functions, optimal strategies exist and thus it follows that there exist a strategy $\pi_{1}$ from $s$ such that $\mathbb{P}_{s}^{\pi_{1}}\left[\operatorname{lr}_{\text {inf }}(\vec{r}) \geq \vec{v}\right]=1$. Since the MDP is strongly connected, the state $s$ can be reached with probability 1 from $s_{0}$ by a strategy $\pi_{2}$. Hence the strategy $\pi_{2}$, followed by the strategy $\pi_{1}$ after reaching $s$, is the witness strategy $\pi^{\prime}$ such that $\mathbb{P}_{s_{0}}^{\pi^{\prime}}\left[\operatorname{lr}_{\text {inf }}(\vec{r}) \geq \vec{v}\right]=1$.

This gives us item 1. of Proposition 5.1 and also immediately implies $\vec{v} \in \operatorname{AcEx}\left(\operatorname{lr}_{\text {inf }}(\vec{r})\right)$. It follows that there are nonnegative values $\bar{x}_{a}$ for all $a \in A$ such that assigning $\bar{x}_{a}$ to $x_{a}$ solves Equations (4.4) and (4.5) of the system $L$ (see Figure 3). Let us assume, w.l.o.g., that $\sum_{a \in A} \bar{x}_{a}=1$.

Lemma 4.3 gives us a memoryless randomized strategy $\zeta$ such that for all BSCCs $D$ of $G^{\zeta}$, all $s \in D \cap S$ and all $a \in D \cap A$ we have that freq $(\zeta, s, a)=\frac{\bar{x}_{a}}{\sum_{a \in D \cap A} \bar{x}_{a}}$. We denote by freq $(\zeta, D, a)$ the value $\frac{\bar{x}_{a}}{\sum_{a \in D \cap A} \bar{x}_{a}}$.

Now we are ready to prove the item 2 of Proposition 5.1. Let us fix $\varepsilon>0$. We obtain $\xi_{\varepsilon}$ by a suitable perturbation of the strategy $\zeta$ in such a way that all actions get positive probabilities and the frequencies of actions change only slightly. There exists an arbitrarily small (strictly) positive solution $x_{a}^{\prime}$ of Equations (4.4) of the system $L$ (it suffices to consider a strategy $\tau$ which always takes the uniform distribution over the actions in every state and then assign freq $\left(\tau, s_{0}, a\right) / N$ to $x_{a}$ for sufficiently large $\left.N\right)$. As the system of Equations (4.4) is linear and homogeneous, assigning $\bar{x}_{a}+x_{a}^{\prime}$ to $x_{a}$ also solves this system and Lemma 4.3 gives us a strategy $\xi_{\varepsilon}$ satisfying freq $\left(\xi_{\varepsilon}, s_{0}, a\right)=\left(\bar{x}_{a}+x_{a}^{\prime}\right) / X$ where $X=\sum_{a^{\prime} \in A} \bar{x}_{a^{\prime}}+x_{a^{\prime}}^{\prime}=1+\sum_{a^{\prime} \in A} x_{a^{\prime}}^{\prime}$. We may safely assume that $\sum_{a^{\prime} \in A} x_{a^{\prime}}^{\prime} \leq \frac{\varepsilon}{2 \cdot M_{r}}$ where $M_{r}=\max _{a \in A} \max _{1 \leq i \leq k}\left|\vec{r}_{i}(a)\right|$. Thus, we obtain

$$
\sum_{a \in A} \operatorname{freq}\left(\xi_{\varepsilon}, s_{0}, a\right) \cdot \vec{r}_{i}(a) \geq \vec{v}_{i}-\varepsilon
$$


by the following sequence of (in)equalities.

$$
\begin{aligned}
\sum_{a \in A} & \operatorname{freq}\left(\xi_{\varepsilon}, s_{0}, a\right) \cdot \vec{r}_{i}(a) \\
& =\sum_{a \in A} \frac{\bar{x}_{a}+x_{a}^{\prime}}{X} \cdot \vec{r}_{i}(a) \\
& =\frac{1}{X} \cdot \sum_{a \in A} \bar{x}_{a} \cdot \vec{r}_{i}(a)+\frac{1}{X} \cdot \sum_{a \in A} x_{a}^{\prime} \cdot \vec{r}_{i}(a)
\end{aligned}
$$

As $G^{\xi_{\varepsilon}}$ is strongly connected, almost all runs $\omega$ of $G^{\xi_{\varepsilon}}$ initiated in $s_{0}$ satisfy

$$
\operatorname{lr}_{\text {inf }}(\vec{r})(\omega)=\sum_{a \in A} \operatorname{freq}\left(\xi_{\varepsilon}, s_{0}, a\right) \cdot \vec{r}(a) \geq \vec{v}-\vec{\varepsilon}
$$

This finishes the proof of item 2 .

Concerning the complexity of computing $\xi_{\varepsilon}$, note that the binary representation of every coefficient in $L$ has only polynomial length. As $\bar{x}_{a}$ 's are obtained as a solution of (a part of) $L$, standard results from linear programming imply that each $\bar{x}_{a}$ has a binary representation computable in polynomial time. The numbers $x_{a}^{\prime}$ are also obtained by solving a part of $L$ and restricted by $\left|\sum_{a^{\prime} \in A} x_{a^{\prime}}^{\prime}\right| \leq \frac{\varepsilon}{2 \cdot M_{r}}$ which allows to compute a binary representation of $x_{a}^{\prime}$ in polynomial time. The strategy $\xi_{\varepsilon}$, defined in the proof of Proposition 5.1, assigns to each action only small arithmetic expressions over $\bar{x}_{a}$ and $x_{a}^{\prime}$. Hence, $\xi_{\varepsilon}$ is computable in polynomial time.

To prove that the problem whether there is some $\xi$ such that $\mathbb{P}_{s_{0}}^{\xi}\left[\operatorname{lr}_{\mathrm{inf}}(\vec{r}) \geq \vec{v}\right]>0$ is decidable in polynomial time, we show that whenever $\vec{v} \in \operatorname{AcEx}\left(\operatorname{lr}_{\text {inf }}(\vec{r})\right)$, then $(1, \vec{v}) \in$ $\operatorname{AcSt}\left(\operatorname{lr}_{\text {inf }}(\vec{r})\right)$. This gives us a polynomial-time algorithm by applying Theorem 4.1, Let $\vec{v} \in \operatorname{AcEx}\left(\operatorname{lr}_{\inf }(\vec{r})\right)$. We show that there is a strategy $\xi$ such that $\mathbb{P}_{s}^{\xi}\left[\operatorname{lr}_{\text {inf }}(\vec{r}) \geq \vec{v}\right]=1$. 
Since $\vec{v} \in \operatorname{AcEx}\left(\operatorname{lr}_{\text {inf }}(\vec{r})\right)$, there are nonnegative rational values $\bar{x}_{a}$ for all $a \in A$ such that assigning $\bar{x}_{a}$ to $x_{a}$ solves Equations (4.4) and (4.5) of the system L. Assume, without loss of generality, that $\sum_{a \in A} \bar{x}_{a}=1$.

Given $a \in A$, let $I_{a}: A \rightarrow\{0,1\}$ be a function given by $I_{a}(a)=1$ and $I_{a}(b)=0$ for all $b \neq a$. For every $i \in \mathbb{N}$, we denote by $\xi_{i}$ a memoryless randomized strategy satisfying $\mathbb{P}_{s}^{\xi_{i}}\left[\operatorname{lr}_{\text {inf }}\left(I_{a}\right) \geq \bar{x}_{a}-2^{-i-1}\right]=1$. Note that for every $i \in \mathbb{N}$ there is $\kappa_{i} \in \mathbb{N}$ such that for all $a \in A$ and $s \in S$ we get

$$
\mathbb{P}_{s}^{\xi_{i}}\left[\inf _{T \geq \kappa_{i}} \frac{1}{T} \sum_{t=0}^{T} I_{a}\left(A_{t}\right) \geq \bar{x}_{a}-2^{-i}\right] \geq 1-2^{-i}
$$

Now let us consider a sequence $n_{0}, n_{1}, \ldots$ of numbers where $n_{i} \geq \kappa_{i}$ and $\frac{\sum_{j<i} n_{j}}{n_{i}} \leq 2^{-i}$ and $\frac{\kappa_{i+1}}{n_{i}} \leq 2^{-i}$. We define $\xi$ to behave as $\xi_{1}$ for the first $n_{1}$ steps, then as $\xi_{2}$ for the next $n_{2}$ steps, then as $\xi_{3}$ for the next $n_{3}$ steps, etc. In general, denoting by $N_{i}$ the sum $\sum_{j<i} n_{j}$, the strategy $\xi$ behaves as $\xi_{i}$ between the $N_{i}$ 'th step (inclusive) and $N_{i+1}$ 'th step (non-inclusive).

Let us give some intuition behind $\xi$. The numbers in the sequence $n_{0}, n_{1}, \ldots$ grow rapidly so that after $\xi_{i}$ is simulated for $n_{i}$ steps, the part of the history when $\xi_{j}$ for $j<i$ were simulated becomes relatively small and has only minor impact on the current average reward (this is ensured by the condition $\frac{\sum_{j<i} n_{j}}{n_{i}} \leq 2^{-i}$ ). This gives us that almost every run has infinitely many prefixes on which the average reward w.r.t. $I_{a}$ is arbitrarily close to $\bar{x}_{a}$ infinitely often. To get that $\bar{x}_{a}$ is also the limit-average reward, one only needs to be careful when the strategy $\xi$ ends behaving as $\xi_{i}$ and starts behaving as $\xi_{i+1}$, because then up to the $\kappa_{i+1}$ steps we have no guarantee that the average reward is close to $\bar{x}_{a}$. This part is taken care of by picking $n_{i}$ so large that the contribution (to the average reward) of the $n_{i}$ steps according to $\xi_{i}$ prevails over fluctuations introduced by the first $\kappa_{i+1}$ steps according to $\xi_{i+1}$ (this is ensured by the condition $\frac{\kappa_{i+1}}{n_{i}} \leq 2^{-i}$ ).

Let us now prove the correctness of the definition of $\xi$ formally. We prove that almost all runs $\omega$ of $G^{\xi}$ satisfy

$$
\liminf _{T \rightarrow \infty} \frac{1}{T} \sum_{t=0}^{T} I_{a}\left(A_{t}(\omega)\right) \geq \bar{x}_{a} .
$$

Denote by $E_{i}$ the set of all runs $\omega=s_{0} a_{0} s_{1} a_{1} \ldots$ of $G^{\xi}$ such that for some $\kappa_{i} \leq d \leq n_{i}$ we have

$$
\frac{1}{d} \sum_{j=N_{i}}^{N_{i}+d} I_{a}\left(a_{j}\right)<\bar{x}_{a}-2^{-i} .
$$

We have $\mathbb{P}_{s_{0}}^{\xi}\left[E_{i}\right] \leq 2^{-i}$ and thus $\sum_{i=1}^{\infty} \mathbb{P}_{s_{0}}^{\xi}\left[E_{i}\right]=\frac{1}{2}<\infty$. By Borel-Cantelli lemma [19], almost surely only finitely many of $E_{i}$ take place. Thus, almost every run $\omega=s_{0} a_{0} s_{1} a_{1} \ldots$ of $G^{\xi}$ satisfies the following: there is $\ell$ such that for all $i \geq \ell$ and all $\kappa_{i} \leq d \leq n_{i}$ we have that

$$
\frac{1}{d} \sum_{j=N_{i}}^{N_{i}+d} I_{a}\left(a_{j}\right) \geq \bar{x}_{a}-2^{-i} .
$$

Consider $T \in \mathbb{N}$ such that $N_{i} \leq T<N_{i+1}$ where $i>\ell$. We need the following inequality

$$
\frac{1}{T} \sum_{t=0}^{T} I_{a}\left(a_{t}\right) \geq\left(\bar{x}_{a}-2^{-i}\right)\left(1-2^{1-i}\right)
$$


which can be proved as follows. First, note that

$$
\frac{1}{T} \sum_{t=0}^{T} I_{a}\left(a_{t}\right) \geq \frac{1}{T} \sum_{t=N_{i-1}}^{N_{i}-1} I_{a}\left(a_{t}\right)+\frac{1}{T} \sum_{t=N_{i}}^{T} I_{a}\left(a_{t}\right)
$$

and that

$$
\frac{1}{T} \sum_{t=N_{i-1}}^{N_{i}-1} I_{a}\left(a_{t}\right)=\frac{1}{n_{i}} \sum_{t=N_{i-1}}^{N_{i}-1} I_{a}\left(a_{t}\right) \cdot \frac{n_{i}}{T} \geq\left(\bar{x}_{a}-2^{-i}\right) \frac{n_{i}}{T}
$$

which gives

$$
\frac{1}{T} \sum_{t=0}^{T} I_{a}\left(a_{t}\right) \geq\left(\bar{x}_{a}-2^{-i}\right) \frac{n_{i}}{T}+\frac{1}{T} \sum_{t=N_{i}}^{T} I_{a}\left(a_{t}\right) .
$$

Now, we distinguish two cases. First, if $T-N_{i} \leq \kappa_{i+1}$, then

$$
\frac{n_{i}}{T} \geq \frac{n_{i}}{N_{i-1}+n_{i}+\kappa_{i+1}}=1-\frac{N_{i-1}+\kappa_{i+1}}{N_{i-1}+n_{i}+\kappa_{i+1}} \geq\left(1-2^{1-i}\right)
$$

and thus, by Equation (5.3),

$$
\frac{1}{T} \sum_{t=0}^{T} I_{a}\left(a_{t}\right) \geq\left(\bar{x}_{a}-2^{-i}\right)\left(1-2^{1-i}\right) .
$$

Second, if $T-N_{i} \geq \kappa_{i+1}$, then

$$
\begin{aligned}
\frac{1}{T} \sum_{t=N_{i}+1}^{T} I_{a}\left(a_{t}\right) & =\frac{1}{T-N_{i}} \sum_{t=N_{i}+1}^{T} I_{a}\left(a_{t}\right) \cdot \frac{T-N_{i}}{T} \\
& \geq\left(\bar{x}_{a}-2^{-i-1}\right)\left(1-\frac{N_{i-1}+n_{i}}{T}\right) \\
& \geq\left(\bar{x}_{a}-2^{-i-1}\right)\left(1-2^{-i}-\frac{n_{i}}{T}\right)
\end{aligned}
$$

and thus, by Equation (5.3),

$$
\begin{aligned}
\frac{1}{T} \sum_{t=0}^{T} I_{a}\left(a_{t}\right) & \geq\left(\bar{x}_{a}-2^{-i}\right) \frac{n_{i}}{T}+\left(\bar{x}_{a}-2^{-i-1}\right)\left(1-2^{-i}-\frac{n_{i}}{T}\right) \\
& \geq\left(\bar{x}_{a}-2^{-i}\right)\left(\frac{n_{i}}{T}+\left(1-2^{-i}-\frac{n_{i}}{T}\right)\right) \\
& \geq\left(\bar{x}_{a}-2^{-i}\right)\left(1-2^{-i}\right)
\end{aligned}
$$

which finishes the proof of Equation (5.2).

Since the sum in Equation (5.2) converges to $\bar{x}_{a}$ as $i$ (and thus also T) goes to $\infty$, we obtain

$$
\liminf _{T \rightarrow \infty} \frac{1}{T} \sum_{t=0}^{T} I_{a}\left(a_{t}\right) \geq \bar{x}_{a}
$$




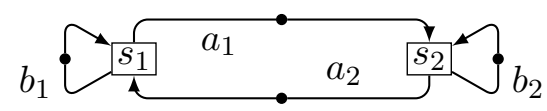

FIGURE 4. MDP showing the need of infinite memory.

The strategy $\xi$ from the proof of Proposition 5.1 required infinite memory. We show that this may indeed be necessary, i.e. it can be the case that $(\nu, \vec{v}) \in \operatorname{AcSt}\left(\operatorname{lr}_{\text {inf }}(\vec{r})\right)$ although there is no finite-memory strategy $\sigma$ satisfying $\mathbb{P}_{s}^{\sigma}\left[\operatorname{lr}_{\text {inf }}(\vec{r}) \geq \vec{v}\right]>\nu$ (and in fact not even finite-memory strategy satisfying $\left.\mathbb{P}_{s}^{\sigma}\left[\operatorname{lr}_{\text {inf }}(\vec{r}) \geq \vec{v}\right]>0\right)$. Consider the MDP from Figure 4, where the reward function $r_{i}$ (for $i \in\{1,2\}$ ) returns 1 for $b_{i}$ and 0 for all other actions. Let $s_{1}$ be the initial vertex. It is easy to see that $(0.5,0.5) \in \operatorname{AcEx}\left(\operatorname{lr}_{\text {inf }}(\vec{r})\right)$ : consider for example a strategy that first chooses both available actions in $s_{1}$ with uniform probabilities, and in subsequent steps chooses self-loops on $s_{1}$ or $s_{2}$ deterministically. From the results presented above we subsequently get that $(1,0.5,0.5) \in \operatorname{AcSt}\left(\operatorname{lr}_{\text {inf }}(\vec{r})\right)$.

On the other hand, let $\sigma$ be arbitrary finite-memory strategy. The Markov chain it induces is by definition finite and for each of its BSCC $C$ we have the following. One of the following then takes place:

- $C$ contains both $s_{1}$ and $s_{2}$. Then by Ergodic theorem for almost every run $\omega$ we have $\operatorname{lr}_{\text {inf }}\left(I_{a_{1}}\right)(\omega)+\operatorname{lr}_{\text {inf }}\left(I_{a_{2}}\right)(\omega)>0$, which means that $\operatorname{lr}_{\text {inf }}\left(I_{b_{1}}\right)(\omega)+\operatorname{lr}_{\text {inf }}\left(I_{b_{2}}\right)(\omega)<1$, and thus necessarily $\operatorname{lr}_{\text {inf }}(\vec{r})(\omega) \nsupseteq(0.5,0.5)$.

- $C$ contains only the state $s_{1}$ (resp. $s_{2}$ ), in which case all runs that enter it satisfy $\operatorname{lr}_{\text {inf }}(\vec{r})(\omega)=(1,0)\left(\right.$ resp. $\left.\operatorname{lr}_{\inf }(\vec{r})(\omega)=(0,1)\right)$.

From the basic results of the theory of Markov chains we get $\mathbb{P}_{s_{1}}^{\sigma}\left[\operatorname{lr}_{\inf }(\vec{r}) \geq(0.5,0.5)\right]=0$.

It is also easy to prove that $\varepsilon$-optimal strategies are not necessarily memoryless pure, as the following lemma shows.

Lemma 5.2. There is an MDP $G$ a vector of reward functions $\vec{r}=\left(r_{1}, r_{2}\right)$, a number $\varepsilon>0$ and a vector $(\nu, \vec{v}) \in \operatorname{AcSt}\left(\operatorname{lr}_{\inf }(\vec{r})\right)$ such that there is no memoryless-pure strategy $\sigma$ satisfying $\mathbb{P}_{s}^{\sigma}\left[\operatorname{lr}_{\inf }(\vec{r}) \geq \vec{v}-\vec{\varepsilon}\right]>\nu-\vec{\varepsilon}$.

Proof. We can reuse $G$ and $\vec{r}$ showing the need of infinite memory for optimal strategies. We let $\nu=1$ and $\vec{v}=(0.5,0.5)$. We have shown that $(\nu, \vec{v}) \in \operatorname{AcSt}\left(\operatorname{lr}_{\inf }(\vec{r})\right)$. Taking e.g. $\varepsilon=0.1$, it is a trivial observation that no memoryless pure strategy satisfies $\mathbb{P}_{s}^{\sigma}\left[\operatorname{li}_{\inf }(\vec{r}) \geq \vec{v}-\vec{\varepsilon}\right]>$ $\nu-\vec{\varepsilon}$.

We are now ready to prove the items B.1, B.3 and B.4. Let $C_{1}, \ldots, C_{\ell}$ be all MECs of $G$. We say that a MEC $C_{i}$ is good for $\vec{v}$ if there is a state $s$ of $C_{i}$ and a strategy $\pi$ satisfying $\mathbb{P}_{s}^{\pi}\left[\operatorname{lr}_{\text {inf }}(\vec{r}) \geq \vec{v}\right]>0$ that never leaves $C_{i}$ when starting in $s$. Using Proposition 5.1 , we can decide in polynomial time whether a given MEC is good for a given $\vec{v}$. Let $\mathcal{C}$ be the union of all MECs good for $\vec{v}$. Then, by Proposition 5.1, there is a strategy $\xi$ such that for all $s \in \mathcal{C}$ we have $\mathbb{P}_{s}^{\xi}\left[\operatorname{lr}_{\text {inf }}(\vec{r}) \geq \vec{v}\right]=1$ and for each $\varepsilon>0$ there is a memoryless randomized strategy $\xi_{\varepsilon}$, computable in polynomial time, such that for all $s \in \mathcal{C}$ we have $\mathbb{P}_{s_{0}}^{\xi_{\varepsilon}}\left[\operatorname{lr}_{\text {inf }}(\vec{r}) \geq \vec{v}-\vec{\varepsilon}\right]=1$.

Consider a strategy $\tau$, computable in polynomial time, which maximizes the probability of reaching $\mathcal{C}$. Denote by $\sigma$ a strategy which behaves as $\tau$ before reaching $\mathcal{C}$ and as $\xi$ afterwards. Similarly, denote by $\sigma_{\varepsilon}$ a strategy which behaves as $\tau$ before reaching $\mathcal{C}$ and as $\xi_{\varepsilon}$ afterwards. Note that $\sigma_{\varepsilon}$ is computable in polynomial time. 
Clearly, $(\nu, \vec{v}) \in \operatorname{AcSt}\left(\operatorname{lr}_{\text {inf }}(\vec{r})\right)$ iff $\mathbb{P}_{s_{0}}^{\tau}[\operatorname{Reach}(\mathcal{C})] \geq \nu$ because $\sigma$ achieves $\vec{v}$ with probability $\mathbb{P}_{s_{0}}^{\tau}[\operatorname{Reach}(\mathcal{C})]$. Thus, we obtain that $\nu \leq \mathbb{P}_{s_{0}}^{\tau}[\operatorname{Reach}(\mathcal{C})] \leq \mathbb{P}_{s_{0}}^{\xi_{\varepsilon}}\left[\operatorname{lr}_{\text {inf }}(\vec{r}) \geq \vec{v}-\vec{\varepsilon}\right]$.

Finally, in order to decide whether $(\nu, \vec{v}) \in \operatorname{AcSt}\left(\operatorname{lr}_{\text {inf }}(\vec{r})\right)$, it suffices to decide whether $\mathbb{P}_{s_{0}}^{\tau}[\operatorname{Reach}(\mathcal{C})] \geq \nu$ in polynomial time.

Now we prove item B.2. Suppose $(\nu, \vec{v})$ is a vector of the Pareto curve. We let $\mathcal{C}$ be the union of all MECs good for $\vec{v}$. Recall that the Pareto curve constructed for expectation objectives is achievable (item A.2). Due to the correspondence between AcSt and AcEx in strongly connected MDPs we obtain the following. There is $\lambda>0$ such that for every MEC $D$ not contained in $\mathcal{C}$, every $s \in D$, and every strategy $\sigma$ that does not leave $D$, it is possible to have $\mathbb{P}_{s}^{\sigma}\left[\operatorname{lr}_{\text {inf }}(\vec{r}) \geq \vec{u}\right]>0$ only if there is $i$ such that $\vec{v}_{i}-\vec{u}_{i} \geq \lambda$, i.e., when $\vec{v}$ is greater than $\vec{u}$ by $\lambda$ in some component. Thus, for every $\varepsilon<\lambda$ and every strategy $\sigma$ such that $\mathbb{P}_{s_{0}}^{\sigma}\left[\operatorname{lr}_{\text {inf }}(\vec{r}) \geq \vec{v}-\vec{\varepsilon}\right] \geq \nu-\varepsilon$ it must be the case that $\mathbb{P}_{s_{0}}^{\sigma}[\operatorname{Reach}(\mathcal{C})] \geq \nu-\varepsilon$. Because for single objective reachability the optimal strategies exist, we get that there is a strategy $\tau$ satisfying $\mathbb{P}_{s_{0}}^{\tau}[\operatorname{Reach}(\mathcal{C})] \geq \nu$, and by using methods similar to the ones of the previous paragraphs we obtain $(\nu, \vec{v}) \in \operatorname{AcSt}\left(\operatorname{lr}_{\text {inf }}(\vec{r})\right)$.

The polynomial-time algorithm mentioned in item B.5 works as follows. First check whether $(\nu, \vec{v}) \in \operatorname{AcSt}\left(\operatorname{lr}_{\text {inf }}(\vec{r})\right)$ and if not, return "no". Otherwise, find all MECs good for $\vec{v}$ and compute the maximal probability of reaching them from the initial state. If the probability is strictly greater than $\nu$, return "no". Otherwise, continue by performing the following procedure for every $1 \leq i \leq k$, where $k$ is the dimension of $\vec{v}$ : Find all MECs $C$ for which there is $\varepsilon>0$ such that $C$ is good for $\vec{u}$, where $\vec{u}$ is obtained from $\vec{v}$ by increasing the $i$-th component by $\varepsilon$ (this can be done in polynomial time using linear programming). Compute the maximal probability of reaching these MECs. If for any $i$ the probability is at least $\nu$, return "no", otherwise return "yes".

The first claim of B.6 follows from Running example (II). We prove that the set $N:=$ $\{\nu \mid(\nu, \vec{v}) \in P\}$, where $P$ is the Pareto curve for $\operatorname{AcSt}\left(\operatorname{lr}_{\text {inf }}(\vec{r})\right)$, is indeed finite. As we already showed, for every fixed $\vec{v}$ there is a union $\mathcal{C}$ of MECs good for $\vec{v}$, and $(\nu, \vec{v}) \in \operatorname{AcSt}\left(\operatorname{lr}_{\inf }(\vec{r})\right)$ iff the $\mathcal{C}$ can be reached with probability at least $\nu$. Hence $|N| \leq 2^{|G|}$, because the latter is an upper bound on a number of unions of MECs in $G$.

To prove the other claims, let $N$ be the set $\{\nu \mid(\nu, \vec{v}) \in P\}$ where $P$ is the Pareto curve for $\operatorname{AcSt}\left(\operatorname{lr}_{\mathrm{inf}}(\vec{r})\right)$.

Let us consider a fixed $\nu \in N$. This gives us a collection $R(\nu)$ of all unions $\mathcal{C}$ of MECs which can be reached with probability at least $\nu$. For a MEC $C$ let $\operatorname{Sol}(C)$ be the set $\operatorname{AcEx}\left(\operatorname{lr}_{\text {inf }}(\vec{r})\right)$ of the MDP given by restricting $G$ to $C$. Further, for every $\mathcal{C} \in R(\nu)$ we set $\operatorname{Sol}(\mathcal{C}):=\bigcap_{C \in \mathcal{C}} \operatorname{Sol}(C)$. Finally, $\operatorname{Sol}(R(\nu)):=\bigcup_{\mathcal{C} \in R(\nu)} \operatorname{Sol}(\mathcal{C})$. From the analysis above we already know that $\operatorname{Sol}(R(\nu))=\left\{\vec{v} \mid(\nu, \vec{v}) \in \operatorname{AcSt}\left(\operatorname{lr}_{\text {inf }}(\vec{r})\right\}\right.$. As a consequence, $(\nu, \vec{v}) \in P$ iff $\nu \in N$ and $\vec{v}$ is maximal in $\operatorname{Sol}(R(\nu))$ and $\vec{v} \notin \operatorname{Sol}\left(R\left(\nu^{\prime}\right)\right)$ for any $\nu^{\prime} \in N, \nu^{\prime}>\nu$. In other words, $P$ is also the Pareto curve of the set $Q:=\{(\nu, \vec{v}) \mid \nu \in N, \vec{v} \in \operatorname{Sol}(R(\nu))\}$. Observe that $Q$ is a finite union of downward closures of bounded convex polytopes, because every $\operatorname{Sol}(\mathcal{C})$ is a bounded convex polytope. Finally, observe that $N$ can be computed using the algorithms for optimizing single-objective reachability. Further, the inequalities defining $\operatorname{Sol}(\mathcal{C})$ can also be computed using our results on AcEx. By a generalised convex polytope we denote a set of points described by a finite conjunction of linear inequalities, which may be both strict and non-strict. 
Claim 5.3. Let $X$ be a generalised convex polytope. The smallest convex polytope containing $X$ is its closure, $\operatorname{cl}(X)$. Moreover, the set $\operatorname{cl}(X) \backslash X$ is a union of some of the facets of $\operatorname{cl}(X)$.

Proof. Let $I$ by the set of inequalities defining $X$, and denote by $I^{\prime}$ the modification of this set where all the inequalities are transformed to non-strict ones. The closure $\operatorname{cl}(X)$ indeed is a convex polytope, as it is described by $I^{\prime}$. Since every convex polytope is closed, if it contains $X$ then it must contain also its closure. Thus $\operatorname{cl}(X)$ is the smallest one containing $X$. Let $\alpha<\beta$ be a strict inequality from $I$. By $I^{\prime}(\alpha=\beta)$ we denote the set $I^{\prime} \cup\{\alpha=\beta\}$. The points of $\operatorname{cl}(X) \backslash X$ form a union of convex polytopes, each one given by the set $I^{\prime}(\alpha=\beta)$ for some $\alpha<\beta \in I$. Thus, it is a union of facets of $c l(X)$.

The following lemma now finishes the proof of B.6:

Lemma 5.4. Let $Q$ be a finite union of bounded convex polytopes, $Q_{1}, \ldots, Q_{m}$. Then its Pareto curve $P$ is a finite union of bounded generalised convex polytopes, $P_{1}, \ldots, P_{n}$. Moreover, if the inequalities describing $Q_{i}$ are given, then the inequalities describing $P_{i}$ can be computed.

Proof. We proceed by induction on the number $m$ of components of $Q$. If $m=0$ then $P=\emptyset$ is clearly a bounded convex polytope easily described by arbitrary two incompatible inequalities. For $m \geq 1$ we denote set $Q^{\prime}:=\bigcup_{i=1}^{m-1} Q_{i}$. By the induction hypothesis, the Pareto curve of $Q^{\prime}$ is some $P^{\prime}:=\bigcup_{i=1}^{n^{\prime}} P_{i}$ where every $P_{i}, 1 \leq i \leq n^{\prime}$ is a bounded generalised convex polytope, described by some set of linear inequalities. Denote by $\operatorname{dom}(X)$ the (downward closed) set of all points dominated by some point of $X$. Observe that $P$, the Pareto curve of $Q$, is the union of all points which either are maximal in $Q_{m}$ and do not belong to $\operatorname{dom}\left(P^{\prime}\right)$ (observe that $\operatorname{dom}\left(P^{\prime}\right)=\operatorname{dom}\left(Q^{\prime}\right)$ ), or are in $P^{\prime}$ and do not belong to $\operatorname{dom}\left(Q_{m}\right)$. In symbols:

$$
P=\left(\text { maximal from } Q_{m} \backslash \operatorname{dom}\left(P^{\prime}\right)\right) \cup\left(P^{\prime} \backslash \operatorname{dom}\left(Q_{m}\right)\right) \text {. }
$$

The set $\operatorname{dom}\left(P^{\prime}\right)$ of all $\vec{x}$ for which there is some $\vec{y} \in P^{\prime}$ such that $\vec{y} \geq \vec{x}$ is a union of projections of generalised convex polytopes - just add the inequalities from the definition of each $P_{i}$ instantiated with $\vec{y}$ to the inequality $\vec{y} \geq \vec{x}$, and remove $\vec{x}$ by projecting. Thus, $\operatorname{dom}\left(P^{\prime}\right)$ is a union of generalised convex polytopes itself. A difference of two generalised convex polytopes is a union of generalised convex polytopes. Thus the set "maximal from $Q_{m} \backslash \operatorname{dom}\left(P^{\prime}\right)$ " is a union of generalised bounded convex polytopes, and for the same reasons so is $P^{\prime} \backslash \operatorname{dom}\left(Q_{m}\right)$.

Finally, let us show how to compute $P$. This amounts to computing the projection, and the set difference. For convex polytopes, efficient computing of projections is a problem studied since the 19th century. One of possible approaches, non-optimal from the complexity point of view, but easy to explain, is by traversing the vertices of the convex polytope and projecting them individually, and then taking the convex hull of those vertices. To compute a projection of a generalised convex polytope $X$, we first take its closure $c l(X)$, and project the closure. Then we traverse all the facets of the projection and mark every facet to which at least one point of $X$ projected. This can be verified by testing whether the inequalities defining the facet in conjunction with the inequalities defining $X$ have a solution. Finally, we remove from the projection all facets which are not marked. Due to Claim 5.3, the difference of the projection of $\operatorname{cl}(X)$ and the projection of $X$ is a union of facets. Every facet from the difference has the property that no point from $X$ is projected to it. Thus we obtained the projection of $X$. 
Computing the set difference of two bounded generalised convex polytopes is easier: Consider we have two polytopes, given by sets $I_{1}$ and $I_{2}$ of inequalities. Then subtracting the second generalised convex polytope from the first is the union of generalised polytopes given by the inequalities $I_{1} \cup\{\alpha \nprec \beta\}$, where $\alpha \prec \beta$ ranges over all inequalities (strict or non-strict) in $I_{2}$.

\section{A Note on Equivalence of Definitions of Strategies}

In this section we argue that the definitions of strategies as functions $(S A)^{*} S \rightarrow \operatorname{dist}(A)$ and as triples $\left(\sigma_{u}, \sigma_{n}, \alpha\right)$ are interchangeable.

Note that formally a strategy $\pi:(S A)^{*} S \rightarrow \operatorname{dist}(A)$ gives rise to a Markov chain $G^{\pi}$ with states $(S A)^{*} S$ and transitions $w^{\sigma(w)(a) \cdot \delta(a)(s)} \underset{\rightarrow}{\rightarrow}$ was for all $w \in(S A)^{*} S, a \in A$ and $s \in S$. Given $\sigma=\left(\sigma_{u}, \sigma_{n}, \alpha\right)$ and a run $w=\left(s_{0}, m_{0}, a_{0}\right)\left(s_{1}, m_{1}, a_{1}\right) \ldots$ of $G^{\sigma}$ denote $w[i]=s_{0} a_{0} s_{1} a_{1} \ldots s_{i-1} a_{i-1} s_{i}$. We define $f(w)=w[0] w[1] w[2] \ldots$

We need to show that for every strategy $\sigma=\left(\sigma_{u}, \sigma_{n}, \alpha\right)$ there is a strategy $\pi:(S A)^{*} S \rightarrow$ $\operatorname{dist}(A)$ (and vice versa) such that for every set of runs $W$ of $G^{\pi}$ we have $\mathbb{P}_{s_{0}}^{\sigma}\left[f^{-1}(W)\right]=$ $\mathbb{P}_{s_{0}}^{\pi}[W]$. We only present the construction of strategies and basic arguments, the technical part of the proof is straightforward.

Given $\pi:(S A)^{*} S \rightarrow \operatorname{dist}(A)$, one can easily define a deterministic-update strategy $\sigma=\left(\sigma_{u}, \sigma_{n}, \alpha\right)$ which uses memory $(S A)^{*} S$. The initial memory element is the initial state $s_{0}$, the next move function is defined by $\sigma(s, w)=\pi(w)$, and the memory update function $\sigma_{u}$ is defined by $\sigma_{u}(a, s, w)=$ was. Reader can observe that there is a naturally defined bijection between runs in $G^{\pi}$ and in $G^{\sigma}$, and that this bijection preserves probabilities of sets of runs.

In the opposite direction, given $\sigma=\left(\sigma_{u}, \sigma_{n}, \alpha\right)$, we define $\pi:(S A)^{*} S \rightarrow \operatorname{dist}(A)$ as follows. Given $w=s_{0} a_{0} \ldots s_{n-1} a_{n-1} s_{n} \in(S A)^{*} S$ and $a \in A$, we denote by $U_{a}^{w}$ the set of all paths in $G^{\sigma}$ that have the form

$$
\left(s_{0}, m_{0}, a_{0}\right)\left(s_{1}, m_{1}, a_{1}\right) \ldots\left(s_{n-1}, m_{n-1}, a_{n_{1}}\right)\left(s_{n}, m_{n}, a\right)
$$

for some $m_{1}, \ldots m_{n}$. We put $\pi(w)(a)=\frac{\mathbb{P}_{s_{0}}^{\sigma}\left[U_{a}^{w}\right]}{\sum_{a^{\prime} \in A^{2}} \mathbb{P}_{s_{0}}^{\sigma}\left[U_{a^{\prime}}^{w}\right]}$. The key observation for the proof of correctness of this construction is that the probability of $U_{a}^{w}$ in $G^{\sigma}$ is equal to probability of taking a path $w$ and then an action $a$ in $G^{\pi}$.

\section{Conclusions}

In this paper we have studied the problem of determining whether for a given MDP there exists a strategy achieving a certain value in each of multiple given limit-average objective functions. We have concentrated on two different interpretations of the functions, namely the expectation objectives and satisfaction objectives, and provided algorithms solving the problem.

The next step in this line of research is to implement and evaluate the algorithms. On the theoretical side, one could further study the problem of existence of a strategy that simultaneously satisfies several expectation objective and satisfaction objectives, or even combine the limit-average functions with different kinds of functions, such as $\omega$-regular objectives or cumulative reward objectives. 
Acknowledgements. The authors thank David Parker and Dominik Wojtczak for initial discussions on the topic. T. Brázdil is supported by the Czech Science Foundation, grant No P202/12/P612. K. Chatterjee is supported by the Austrian Science Fund (FWF) Grant No P 23499-N23; FWF NFN Grant No S11407-N23 (RiSE); ERC Start grant (279307: Graph Games); Microsoft faculty fellows award. V. Forejt is supported by a Royal Society Newton Fellowship and EPSRC project EP/J012564/1.

\section{REFERENCES}

[1] E. Altman. Constrained Markov Decision Processes (Stochastic Modeling). Chapman \& Hall/CRC, 1999.

[2] S. Boyd and L. Vandenberghe. Convex Optimization. Cambridge Univ. Press, 2004.

[3] T. Brázdil, V. Brožek, K. Chatterjee, V. Forejt, and A. Kučera. Two views on multiple mean-payoff objectives in markov decision processes. In LICS, pages 33-42. IEEE Computer Society, 2011.

[4] T. Brázdil, V. Brožek, and K. Etessami. One-counter stochastic games. In K. Lodaya and M. Mahajan, editors, FSTTCS, volume 8 of LIPIcs, pages 108-119. Schloss Dagstuhl - Leibniz-Zentrum für Informatik, 2010.

[5] T. Brázdil, V. Brožek, V. Forejt, and A. Kučera. Reachability in recursive markov decision processes. Inf. Comput., 206(5):520-537, 2008.

[6] K. Chatterjee. Concurrent games with tail objectives. Theor. Comput. Sci., 388:181-198, December 2007.

[7] K. Chatterjee. Markov decision processes with multiple long-run average objectives. In V. Arvind and S. Prasad, editors, FSTTCS, volume 4855 of Lecture Notes in Computer Science, pages 473-484. Springer, 2007.

[8] K. Chatterjee, R. Majumdar, and T. A. Henzinger. Markov decision processes with multiple objectives. In B. Durand and W. Thomas, editors, STACS, volume 3884 of Lecture Notes in Computer Science, pages 325-336. Springer, 2006.

[9] C. Courcoubetis and M. Yannakakis. Markov decision processes and regular events. Automatic Control, IEEE Transactions on, 43(10):1399-1418, Oct. 1998.

[10] K. Etessami, M. Kwiatkowska, M. Vardi, and M. Yannakakis. Multi-objective model checking of Markov decision processes. LMCS, 4(4):1-21, 2008.

[11] J. Filar and K. Vrieze. Competitive Markov Decision Processes. Springer-Verlag, 1997.

[12] V. Forejt, M. Z. Kwiatkowska, G. Norman, D. Parker, and H. Qu. Quantitative multi-objective verification for probabilistic systems. In P. A. Abdulla and K. R. M. Leino, editors, TACAS, volume 6605 of Lecture Notes in Computer Science, pages 112-127. Springer, 2011.

[13] H. Gimbert and F. Horn. Solving simple stochastic tail games. In M. Charikar, editor, SODA, pages 847-862. SIAM, 2010.

[14] J. Koski. Multicriteria truss optimization. In W. Stadler, editor, Multicriteria Optimization in Engineering and in the Sciences. Plenum Press, 1988.

[15] J. R. Norris. Markov chains. Cambridge University Press, 1998.

[16] G. Owen. Game Theory. Academic Press, 1995.

[17] C. H. Papadimitriou and M. Yannakakis. On the approximability of trade-offs and optimal access of web sources. In FOCS, pages 86-92. IEEE Computer Society, 2000.

[18] M. Puterman. Markov Decision Processes. John Wiley and Sons, 1994.

[19] H. Royden. Real analysis. Prentice Hall, 3rd edition, 12 Feb. 1988.

[20] R. Szymanek, F. Catthoor, and K. Kuchcinski. Time-energy design space exploration for multi-layer memory architectures. In DATE, pages 318-323. IEEE Computer Society, 2004.

[21] P. Yang and F. Catthoor. Pareto-optimization-based run-time task scheduling for embedded systems. In R. Gupta, Y. Nakamura, A. Orailoglu, and P. H. Chou, editors, CODES+ISSS, pages 120-125. ACM, 2003 . 